\title{
Self-Consistent Approach to Global Charge Neutrality in Electrokinetics: A Surface Potential Trap Model
}

\author{
Li Wan, ${ }^{1}$ Shixin Xu, ${ }^{3,4}$ Maijia Liao, ${ }^{1}$ Chun Liu, ${ }^{4, *}$ and Ping Sheng ${ }^{1,2, \dagger}$ \\ ${ }^{1}$ Department of Physics, Hong Kong University of Science and Technology, \\ Clear Water Bay, Kowloon, Hong Kong, China \\ ${ }^{2}$ Institute for Advanced Study, Hong Kong University of Science and Technology, \\ Clear Water Bay, Kowloon, Hong Kong, China \\ ${ }^{3}$ School of Mathematical Sciences, Soochow University, Suzhou, China \\ ${ }^{4}$ Department of Mathematics, Pennsylvania State University, University Park, Pennsylvania 16802, USA \\ (Received 8 April 2013; revised manuscript received 4 September 2013; published 18 March 2014)
}

In this work, we treat the Poisson-Nernst-Planck (PNP) equations as the basis for a consistent framework of the electrokinetic effects. The static limit of the PNP equations is shown to be the charge-conserving Poisson-Boltzmann (CCPB) equation, with guaranteed charge neutrality within the computational domain. We propose a surface potential trap model that attributes an energy cost to the interfacial charge dissociation. In conjunction with the CCPB, the surface potential trap can cause a surface-specific adsorbed charge layer $\sigma$. By defining a chemical potential $\mu$ that arises from the charge neutrality constraint, a reformulated CCPB can be reduced to the form of the Poisson-Boltzmann equation, whose prediction of the Debye screening layer profile is in excellent agreement with that of the Poisson-Boltzmann equation when the channel width is much larger than the Debye length. However, important differences emerge when the channel width is small, so the Debye screening layers from the opposite sides of the channel overlap with each other. In particular, the theory automatically yields a variation of $\sigma$ that is generally known as the "charge regulation" behavior, attendant with predictions of force variation as a function of nanoscale separation between two charged surfaces that are in good agreement with the experiments, with no adjustable or additional parameters. We give a generalized definition of the $\zeta$ potential that reflects the strength of the electrokinetic effect; its variations with the concentration of surface-specific and surfacenonspecific salt ions are shown to be in good agreement with the experiments. To delineate the behavior of the electro-osmotic (EO) effect, the coupled PNP and Navier-Stokes equations are solved numerically under an applied electric field tangential to the fluid-solid interface. The EO effect is shown to exhibit an intrinsic time dependence that is noninertial in its origin. Under a step-function applied electric field, a pulse of fluid flow is followed by relaxation to a new ion distribution, owing to the diffusive counter current. We have numerically evaluated the Onsager coefficients associated with the EO effect, $L_{21}$, and its reverse streaming potential effect, $L_{12}$, and show that $L_{12}=L_{21}$ in accordance with the Onsager relation. We conclude by noting some of the challenges ahead.

DOI: 10.1103/PhysRevX.4.011042

\section{INTRODUCTION}

Electrokinetics (EK) is a classic subject that dates back nearly two centuries [1]. It involves the interaction of ions in the fluid with the solid surface, and their dynamics under an applied electric field tangential to the fluid-solid interface. The EK effect includes four main topics: electroosmosis (EO), streaming potential (SP), electrophoresis,

\footnotetext{
*liu@math.psu.edu

${ }^{\dagger}$ sheng@ust.hk
}

Published by the American Physical Society under the terms of the Creative Commons Attribution 3.0 License. Further distribution of this work must maintain attribution to the author(s) and the published article's title, journal citation, and DOI.
Subject Areas: Interdisciplinary Physics, Soft Matter, Statistical Physics and sedimentation potential [2-5]. In the past two decades, the EK effect has experienced a strong revival mainly owing to its various potential applications, e.g., an EO pump with no moving parts that can propel electrolytes and particles in the microscale [6-23], as well as manipulate biological cells [24-31]. With the advent of microfluidic technology [32-45], many EK effect-based microfluidic devices have been fabricated, providing not only the opportunity to study the basic mechanisms of the EK effect, but also the push to extend its applications into many diverse interdisciplinary areas.

It is well known that the formation of the electric double layer (EDL) at the fluid-solid interface, as initially studied by Gouy and Chapman [2,46-68], is the crucial element of the EK effect. A thin layer of charge is generated at the 
interface, thereby leading to the formation of a diffuse screening layer of mobile counter ions, usually denoted the Debye layer. An important fact is that there is a net amount of charge in the Debye layer; hence, in the presence of an externally applied electric field parallel to the interface, there can be a body force to drag the fluid along. For the thin layer of surface charge attached to the interface, it can experience a force in the opposite direction. However, either through the hydrodynamic no-slip boundary condition or physical attachment to the solid, its movement is either small or nonexistent. That layer is usually denoted the "Stern layer" [63], which is usually further divided into a layer formed by surface-specific adsorbed ions, plus a layer adsorbed from the bulk, with its thickness accounted for by the ion size [68]. The dividing plane between the two is usually denoted the inner Helmholtz plane (iHp), whereas the plane separating the Stern layer and the diffuse layer is denoted the outer Helmholtz plane (oHp).

The mechanism of EDL has been reviewed by several authors [69-72]. In the standard mathematical description of the EDL model, the Poisson-Boltzmann (PB) equation is used to delineate the charge and potential distributions in the Debye layer. Traditionally, in solving the PB equation, a constant $\zeta$ (zeta) potential is assumed at the boundary. By coupling the solution of the PB equation to the Navier-Stokes (NS) equation, the electro-osmotic effect, i.e., that an electric field can lead to fluid flow, is easily modeled [48-67].

There are some well-known issues associated with the PB equation and the related EK effect description. First of all, the $\mathrm{PB}$ equation dictates a reference (electrical) potential to be associated with local charge neutrality within the fluid domain (see below). This can become an issue when the separation between two charged surfaces is on the nanoscale, i.e., when there is an overlap between the EDLs generated from the two opposite surfaces so that there is no local charge neutrality anywhere in the fluid domain. Second, since charge neutrality is not preserved in the computational domain, overall charge neutrality requires auxiliary conditions to be put in by hand; i.e., compensating charges need to be placed outside the computational domain to restore overall charge neutrality. The resulting treatment is therefore not mathematically self-contained. This can be a problem in treating nanoscale or time-varying electrokinetics, for example, since the interfacial charge layer would necessarily respond to the local fluid conditions, either as a function of time or as a function of separation between two charged surfaces, thereby requiring constant hands-on corrections for achieving charge neutrality. Even if such adjustments can be done, they may not necessarily be consistent with the principle of free-energy minimization. Third, by treating the $\zeta$ potential as a constant input boundary condition, one ignores the fact that it has been observed to vary with bulk ion density, and hence, theoretically, it should be a derived (calculated) quantity rather than an input. Such correlated variation also emphasizes the need for having both the ions and counterions be within the computational domain so that their interaction can be treated in a mathematically self-consistent manner. Fourth, the timevarying $\mathrm{EO}$ effect has been widely observed but rarely reported or addressed [73,74]. In particular, no theoretical study has been carried out to investigate whether there can be an intrinsic (noninertial) time dependence to the EO effect, even though from physical considerations the inevitable existence of diffusive counterion currents must give rise to a relaxation process.

More recently, there have been a number of experiments showing that when the fluid-channel width, i.e., the planar separation, is in the nanometer regime (smaller than the Debye length), the predictions of the classical theory need to be further examined [75-82], owing to the different predictions offered by the constant potential boundary condition (CP) and the constant (surface) charge boundary condition (CC) in this regime. Experimental evidences, mainly obtained from atomic-force-microscope measurements, have indicated a variation of the surface charge density when the channel width decreases below the Debye length, which does not agree with the predictions resulting from either the $\mathrm{CP}$ or the $\mathrm{CC}$ boundary condition. This discrepancy is generally attributed to the "charge regulation" phenomenon, denoting the fact that the interfacial charge layer must vary as a function of local environment. Theoretically, the experimental results can be interpreted by using a parameter that interpolates the surface charge density implied by the $\mathrm{CC}$ and $\mathrm{CP}$ boundary conditions [83-86]. However, the physical meaning of the interpolation parameter is not clear.

Such developments, in conjunction with the well-known problems stated above, motivate us to reformulate the mathematical theory by starting from a rigorous basisthe Poisson-Nernst-Planck (PNP) equations together with the NS equation-as an attempt for deriving a consistent description of the EDL and its dynamics under the constraint of overall charge neutrality. Since the description of the EDL and the EK effect necessarily involves a fluid channel bounded by solid surfaces, in this work we choose the geometry of a cylindrical fluid channel with radius $a$ in most of our considerations. The exception is in the evaluation of force between two charged surfaces, which must involve a planar surface as necessitated by the experimental geometry. Also, an important concept used in our mathematical modeling is the "computational domain." It is defined as the spatial region delineated by the boundary on which the mathematical boundary condition is imposed. It is also the spatial region in which the relevant partial differential equations apply.

In what follows, Sec. II introduces the PNP equations with the PB model presented as their static limit. The physical picture of Gouy-Chapman is briefly reviewed. We show in Sec. III that the static limit of the PNP equations is given by the charge-conserved Poisson Boltzmann (CCPB) 
equation, which guarantees overall charge neutrality within the computational domain. In Sec. IV, we propose a surface potential trap model to be used in conjunction with the CCPB or the PNP equations. This model enables charge separation within the computational domain and the consequent formation of a surface-specific adsorbed charge density $\sigma$ [87]. In Sec. IV, we show that in the presence of charge separation, the charge neutrality constraint leads to the definition of a chemical potential $\mu$ for the ions. The CCPB equation can then be reduced to the form of the PB equation, with $\mu$ and $\sigma$ determined self-consistently within the same framework. The $\zeta$ potential, being responsible for the EK effect and hence requiring the existence of a nonuniform electrical potential arising from charge separation, is defined as the volume-averaged potential variation; its variations with the concentration of surface-specific (i.e., $\mathrm{pH}$ values) and nonspecific salt ions (i.e., $\mathrm{pC}$ values) are shown to be in good agreement with the experiments in Sec. V. In particular, we show that the predictions of our model and those from the classical PB equation agree to a high degree of accuracy when the fluid-channel width is large. However, important differences emerge when the fluid-channel width decreases below the Debye screening length. In particular, the variation of $\sigma$ in this regime, which is the focus of the charge regulation phenomena, emerges naturally as a prediction of the theory without any additional parameters. In Sec. VI, we show that attendant with the $\sigma$ variation, the theory predicts a force between two charged surfaces that is in good agreement with the experiments. In Sec. VII, we address the EO effect and its (noninertial) time variation. Under a step-function driving field, the EO effect is manifest as a pulse of fluid flow followed by relaxation to a smaller flux, implying an intrinsic time dependence arising from the tendency to establish a new equilibrium, owing to the diffusive countercurrent. In Sec. VIII, we numerically evaluate the Onsager coefficients associated with the EO effect, $L_{21}$, and its reverse SP effect, $L_{12}$. The results show that our model satisfies the Onsager relation $L_{21}=L_{12}$. We conclude in Sec. IX with a summary of the main points, and note the challenges ahead.

\section{POISSON-NERNST-PLANCK EQUATIONS AND THEIR STATIC LIMIT}

\section{A. Poisson-Nernst-Planck equations}

In a charge-neutral fluid with a given density of positive ions $p(\boldsymbol{x})$ and negative ions $n(\boldsymbol{x})$, where $\boldsymbol{x}$ denotes the spatial coordinate, the overall spatial average of both $p$ and $n$ must be the same, denoted by $n^{o}$. The dynamics of the ions and their interaction should satisfy the charge continuity equation and the Poisson equation. This is expressed in a rigorous manner by the PNP equations [88-93]:

$$
\frac{d n}{d t}+\nabla \cdot \boldsymbol{J}_{n}=0
$$

$$
\begin{gathered}
\frac{d p}{d t}+\nabla \cdot \boldsymbol{J}_{p}=0 \\
\boldsymbol{J}_{n}=-D_{n}\left(\nabla n-\frac{z e}{k_{B} T} n \nabla \varphi\right), \\
\boldsymbol{J}_{p}=-D_{p}\left(\nabla p+\frac{z e}{k_{B} T} p \nabla \varphi\right), \\
\nabla^{2} \varphi=-\frac{z e(p-n)}{\varepsilon} .
\end{gathered}
$$

Here, the parameter $z$ denotes the valence of the ions (taken to be 1 in this work), $e$ is the electronic charge, $\varepsilon$ the dielectric constant of the liquid, $k_{B}$ the Boltzmann constant, and $T=300 \mathrm{~K}$ in this work. $D_{n}\left(D_{p}\right)$ is the diffusion coefficient for negative (positive) ions, which is related to the ionic mobility $\mu_{n}\left(\mu_{p}\right)$ through the Einstein relation: $D_{n(p)} / \mu_{n(p)}=k_{B} T / e, \boldsymbol{J}_{n}\left(\boldsymbol{J}_{p}\right)$ is the negative (positive) ion flux; here each of the two ion currents is seen to comprise the sum of two terms - one for the diffusive flux and the other for the drift (or convective) flux. We shall assume that $D_{p}=$ $D_{n}=D$ in this work. Equations (1a)-(1d) describe the charge continuity condition for both the positive and negative ions, while Eq. (1e) is the Poisson equation relating the net ion charge density to the electrical potential $\varphi$; its form results from the minimization of the total electrical energy of the system. The PNP equations can be solved numerically; an analytical solution to the one-dimensional PNP equations was proposed only recently [94-97]. The PNP equations were used to study ion transport dynamics [98-103]; here, they are regarded as the basis of electrokinetics when coupled with the proper fluid-solid interfacial boundary conditions.

In this work, we treat the simplified problem in which the system is electrically neutral with an overall ion density that is a constant, i.e., $\int_{V} p(\boldsymbol{x}) d \boldsymbol{x}=\int_{V} n(\boldsymbol{x}) d \boldsymbol{x}=n^{o} V$, where $V$ denotes the volume of the system, and the ions are represented by point particles, each carrying a single electronic charge, with no chemical distinctions. An exception is made with respect to the distinction between the ions that can participate in the surface-specific adsorption at the fluid-solid interface and the non-surface-specific ions that do not adsorb onto the fluid-solid interface (Sec. IV). The conditions of electrical neutrality and constant ion density are noted to be easily compatible with the PNP equations and the relevant boundary conditions.

For the description of the EK effect, the PNP equations must be coupled to the Navier-Stokes (NS) equation that governs the hydrodynamics of fluid flow:

$$
\rho_{m}\left(\frac{\partial \boldsymbol{u}}{\partial t}+\boldsymbol{u} \cdot(\nabla \boldsymbol{u})\right)=-\nabla P+\eta \nabla^{2} \boldsymbol{u}-e(p-n) \nabla \varphi,
$$




$$
\nabla \cdot \boldsymbol{u}=0
$$

where $\rho_{m}$ is the fluid mass density, $\boldsymbol{u}$ denotes fluid velocity, $P$ denotes pressure, and $\eta$ is the shear viscosity. Equation $(1 \mathrm{~g})$ is the incompressibility condition of the fluid. When $\boldsymbol{u} \neq 0, d n / d t$ and $d p / d t$ in Eqs. (1a) and (1b) should be understood to denote, respectively, $\partial n / \partial t+$ $\boldsymbol{u} \cdot(\nabla n)$ and $\partial p / \partial t+\boldsymbol{u} \cdot(\nabla p)$.

Some of the kinematic boundary conditions for the PNP and NS equations may be easily stated as follows. At the fluid-solid interface, we should have $\boldsymbol{u}=0$, and $\boldsymbol{J}_{n} \cdot \boldsymbol{n}=0$, $\boldsymbol{J}_{p} \cdot \boldsymbol{n}=0$, where $\boldsymbol{n}$ denotes the interfacial normal. These conditions guarantee the conservation of both $p$ and $n$ and hence the overall charge neutrality. The electrical boundary conditions at the fluid-solid interface are the most important since they give rise to the EDL and hence the electrokinetic phenomena. Traditionally, this can be either the Dirichlet-type boundary condition in which a constant potential is specified, or a Neumann-type boundary condition in which the normal electric field is given. In order to clarify the physical underpinnings of the relevant boundary conditions, in what follows we first treat the static limit of the PNP equations, i.e., the time-independent, zero-current $\left(\boldsymbol{J}_{n}, \boldsymbol{J}_{p}, \boldsymbol{u}=0\right)$ solution, for the purpose of delineating the EDL. This is done in two steps-first by showing how the PB equation can be obtained from the static limit of the PNP equations as well as the inconsistencies that can arise, followed by a simple resolution of the inconsistencies with the attendant implications.

\section{B. Static limit of the PNP equations-the Poisson-Boltzmann model}

The PB equation can be obtained from the PNP equations by setting $\boldsymbol{J}_{n}, \boldsymbol{J}_{p}, \boldsymbol{u}=0$. In that limit, we have

$$
\begin{aligned}
& \nabla n-\frac{e}{k_{B} T} n \nabla \varphi=0, \\
& \nabla p+\frac{e}{k_{B} T} p \nabla \varphi=0 .
\end{aligned}
$$

Equations (2a) and (2b) can be integrated to yield

$$
\begin{aligned}
& n=\alpha \exp \left[+e \varphi / k_{B} T\right], \\
& p=\beta \exp \left[-e \varphi / k_{B} T\right] .
\end{aligned}
$$

In other words, the Boltzmann distribution of the ionic densities is the result of detailed balance between the drift current and the diffusive countercurrent. Here, $\alpha$ and $\beta$ are the integration constants. By setting $\alpha=\beta=n^{\infty}$, where $n^{\infty}$ denotes the average ion density in the bulk limit, and substituting this solution into the Poisson equation, $\nabla^{2} \varphi=-e(p-n) / \varepsilon$, we obtain

$$
\rho=e(p-n)=-2 e n^{\infty} \sinh \left(\frac{e \varphi}{k_{B} T}\right)
$$

and

$$
\nabla^{2} \bar{\varphi}=\frac{1}{\lambda_{D}^{2}} \sinh (\bar{\varphi})
$$

where $\bar{\varphi}=e \varphi / k_{B} T$, with $\lambda_{D}=\sqrt{\varepsilon k_{B} T /\left(2 e^{2} n^{\infty}\right)}$ being the Debye length. Here, the distinction between $n^{\infty}$ and $n^{o}$ is necessitated by the consideration of surface charge density, to be detailed below, which can make the two quantities differ in the regime of finite or small fluid channels. Equation (3b) is known as the Poisson-Boltzmann equation; it represents the traditional starting point of a mathematical treatment of the diffuse screening layer that was first proposed by Gouy and Chapman. The PB model consists of Eq. (3b) together with the required boundary condition(s) for its solution. There are two usual types of boundary conditions. (1) The Dirichlet-type boundary condition is where a constant value of the electrical potential $(\mathrm{CP})$, generally denoted the zeta $(\zeta)$ potential, is specified at the fluid-solid interface. This is supplemented by the condition $\partial_{\perp} \varphi=0$ at the center of the fluid channel (or at infinity), where the subscript $\perp$ denotes the spatial derivative to be normal to the channel axis. (2) The Neumann-type boundary condition is where a constant value of the normal electric field is specified at the fluidsolid interface, plus the same condition at the channel center (or infinity). This is sometimes also denoted the Gouy-Chapman boundary condition, or the constant charge boundary condition (CC) [46,52-58].

It should be especially noted that the condition $\alpha=\beta=n^{\infty}$, together with Eqs. (2c) and (2d), implies that local charge neutrality is directly linked with the condition of $\varphi=0$. Hence, in Eq. (3b), the potential has an absolute reference, located in the fluid where there is local charge neutrality. When this cannot be achieved within a specific model problem, e.g., in a nanoscale fluid channel, it is usually necessary to invoke an infinite reservoir, with guaranteed charge neutrality, so that the value of the $\zeta$ potential can be well defined relative to such a reference.

With a constant $\zeta$ potential at the fluid-solid interface, the solution to Eq. (3b) is known to be an exponential-type decay of the electrical potential, with a decay length given by $\lambda_{D}$. For water with $n^{\infty}=950 / \mu \mathrm{m}^{3} \quad(\mathrm{pH}=8.2)$, $\lambda_{D}=0.24 \mu \mathrm{m}$. In order to facilitate discussion, in what follows we consider a cylindrical channel with radius $a$. Unless otherwise specified, the cylindrical channel is assumed to be either infinite in length or long enough so that the end effects can be ignored. In this case, the translational invariance along the channel axis implies that global charge neutrality is equivalent to charge neutrality at every cross section of the channel. 
For a channel with $a \rightarrow \infty$, it is generally possible to associate the center of the channel with charge neutrality, and hence $\varphi=0$. A constant, nonzero $\zeta$ potential at the cylindrical boundary therefore implicitly implies a potential difference between the center and the boundary and hence a nonzero normal electric field. This is consistent with the fact that the solution to Eq. ( $3 b$ ) must have a net charge [see Eq. (4) below], simply obtainable by integrating the righthand side of Eq. (3a), with the solution $\varphi$ profile, from the center (where $\varphi=0$ ) to the boundary (where $\varphi=\zeta$ ). This net charge will generate a normal electric field as required by the Gauss law.

\section{Physical picture of Gouy-Chapman}

As the PB equation with a constant $\zeta$ potential is seen to violate overall charge neutrality within the computational domain, the usual way of fixing the problem is to add a uniform surface charge layer just outside the computational domain, which can exactly cancel the net charge generated by the solution of the PB equation. Since a layer of uniform surface charge does not give rise to any interior electric field, such an addition will not affect the solution profile. This is the Gouy-Chapman (CC) approach, which specifies the normal electric field at the boundary that is equivalent to the net charge in the Debye layer, equivalent in magnitude to a surface charge density placed just outside the computational domain. It is therefore clear that the two boundary conditions, the Dirichlet type (CP) and the Neumann type (CC), are essentially equivalent and will give rise to identical screening layer profiles, provided the supplemental Gouy-Chapman surface charge density $s^{(\mathrm{GC})}$ is related to $\zeta$ by the following relation in the $\lambda_{D} / a \rightarrow 0$ limit:

$$
s^{(\mathrm{GC})}=4 e n^{\infty} \lambda_{D} \sinh \left(\frac{e \zeta}{2 k_{B} T}\right)+\mathcal{O}\left(\lambda_{D} / a\right) .
$$

It is seen that besides $n^{\infty}$, the boundary value $\zeta$ also plays a role in determining the magnitude (and sign) of the surface charge density. In Fig. 1, we give a schematic illustration of the ion distribution.

While the Dirichlet and Neumann boundary conditions are essentially equivalent when $a \gg \lambda_{D}$, we shall see below that as $a$ decreases below $\lambda_{D}$, the two boundary conditions can yield different predictions if their respective boundary values remain fixed [Fig. 3(c)]. This is because the center of the fluid channel can no longer remain at zero potential when the two Debye layers overlap. Hence, the integration of the right-hand side of the $\mathrm{PB}$ equation, representing the net charge in the fluid, has to decrease if the boundary potential value remains fixed at $\zeta$ (since the potential difference between the center and the boundary decreases). From the Guy-Chapman picture, the corresponding surface charge density must also decrease so as to preserve overall charge neutrality. Hence, the $\mathrm{CP}$ boundary condition implies a decreasing surface charge density when the

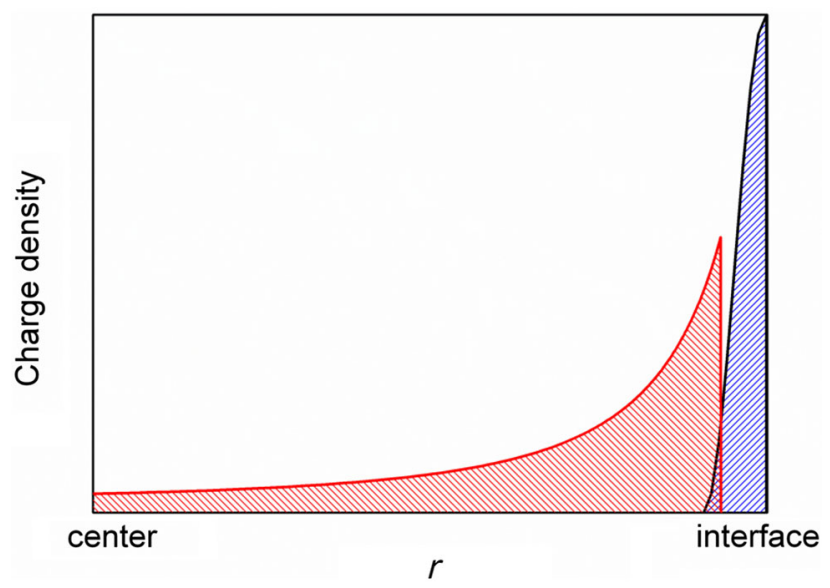

FIG. 1. A schematic illustration of the charge density distribution as a function of the radial coordinate $r$. Here, the blue shading indicates the trapped negative surface ions, significantly exaggerated in thickness for the purpose of illustration, and red shading means the positive counterions that are dominant in the Debye screening layer. The boundary condition of the PB equation is noted to be defined at the interface between the blue and red shadings, i.e., inside the computational domain of the charge-conserving Poisson-Boltzmann equation.

channel width decreases, whereas the CC boundary condition has a fixed surface charge density by definition. Which one is correct? Or are both incorrect? Experimental evidence points to the latter. A significant advantage of the present work, shown below, is that it accurately reproduces the traditional results when the channel width is large, while the predictions for the nanoscale channel width can also agree with the experimental observations without additional or adjustable parameters.

EO flow can result from coupling the $\mathrm{PB}$ equation to the NS equation. This is shown in Appendix A. We also show that the Onsager relation holds for the PB equation under the traditional approximations.

\section{THE CHARGE-CONSERVING POISSON BOLTZMANN EQUATION}

\section{A. Inconsistencies between the PB equation and the PNP equations}

There are two inconsistencies between the PNP equations and the PB equation. First, since the electrical potential in the PNP equations appears only in the form of $\nabla \varphi$, it follows that the solution must be independent of any constant reference potential, i.e., the PNP equations do not need an absolute potential reference as in the case of the $\mathrm{PB}$ equation. A second inconsistency lies in the charge conservation that is inherent in the PNP equations, as pointed out earlier, but there is no such built-in feature in the $\mathrm{PB}$ equation. Even though the overall charge conservation can be restored by adding a supplemental surface charge density as in the Gouy-Chapman approach, 
the PNP equations inform us that charge conservation should hold within the computational domain so that the surface charge density constitutes an integral part of the system that is actively coupled to the bulk.

\section{B. Charge-conserving Poisson-Boltzmann equation}

The two inconsistencies pointed out above can be simply resolved by observing that, from Eqs. (2c) and (2d), the overall charge-neutrality condition dictates

$$
\alpha \int d x \exp \left[+e \varphi / k_{B} T\right]=n^{o} V=\beta \int d x \exp \left[-e \varphi / k_{B} T\right]
$$

which easily follows from $\boldsymbol{J}_{n} \cdot \boldsymbol{n}=0, \boldsymbol{J}_{p} \cdot \boldsymbol{n}=0$ at the sample boundary, plus Eqs. (1a) and (1b). Hence, we see that $\alpha \neq \beta$ in general, in contrast to the previous assumption $\left(\alpha=\beta=n^{\infty}\right)$ that led to the PB equation. From Eq. (5a), it follows that

$$
\begin{aligned}
& n=n^{o} \frac{\exp \left[+e \varphi / k_{B} T\right]}{\frac{1}{V} \int d \boldsymbol{x} \exp \left[+e \varphi / k_{B} T\right]}, \\
& p=n^{o} \frac{\exp \left[-e \varphi / k_{B} T\right]}{\frac{1}{V} \int d \boldsymbol{x} \exp \left[-e \varphi / k_{B} T\right]} .
\end{aligned}
$$

By substituting the above expressions into the Poisson equation, we obtain the following integral-differential equation for a cylindrical channel with radius $a$ :

$$
\begin{aligned}
\frac{1}{r} \frac{\partial}{\partial r}\left(r \frac{\partial \varphi}{\partial r}\right)= & \frac{e a^{2} n^{o}}{2 \varepsilon}\left[\frac{\exp \left(e \varphi / k_{B} T\right)}{\int_{0}^{a} r \exp \left(e \varphi / k_{B} T\right) d r}\right. \\
& \left.-\frac{\exp \left(-e \varphi / k_{B} T\right)}{\int_{0}^{a} r \exp \left(-e \varphi / k_{B} T\right) d r}\right] .
\end{aligned}
$$

This charge-conserving Poisson-Boltzmann (CCPB) equation has been derived previously [104], together with its numerical solution algorithm. Here, we emphasize and point out its physical implications, i.e., consistency with the PNP equations and its relevance to electrokinetics.

It is easy to see that both inconsistencies raised previously are easily resolved. For the reference potential issue, an additive $\varphi_{o}$ would give rise to multiplicative factors, $\exp \left( \pm e \varphi_{o} / k_{B} T\right)$, that are present simultaneously in both the numerator and the denominator on the right-hand side of Eq. (6) and hence cancel. As for charge conservation, the derivation of CCPB itself has incorporated this feature, which is evident from the fact that the volume integral on the right-hand side of Eq. (6) (which represents the net charge density) yields zero.

In contrast to the $\mathrm{PB}$ equation, the $\mathrm{CCPB}$ equation cannot yield an EDL solution with a constant potential boundary condition. This is perhaps clear from the fact that the CCPB is insensitive to a constant reference potential, as pointed out above; hence, a constant potential boundary condition is equivalent to a zero potential boundary condition, which would clearly yield nothing.

In addition to the above, the CCPB cannot yield an EDL with the Neumann-type boundary condition either. This can be seen as follows. Multiply both sides of Eq. (6) by $2 \pi r d r$ and integrate from 0 to $a$. The right-hand side of the equation yields zero after the integration because of the charge-neutrality condition. The left-hand side yields $\left.r(\partial \varphi / \partial r)\right|_{r=a}-\left.r(\partial \varphi / \partial r)\right|_{r=0}=\left.r(\partial \varphi / \partial r)\right|_{r=a}$. Hence, $\partial \varphi /\left.\partial r\right|_{r=a}$ must be zero. If one specifies a Neumann boundary condition with $\partial \varphi /\left.\partial r\right|_{r=a} \neq 0$, then it will be inconsistent with the CCPB equation and there will be no solution. However, if $\partial \varphi /\left.\partial r\right|_{r=a}=0$ is applied, then the solution is a constant $\varphi$ throughout; hence, again, no EDL can be generated.

From these simple deductions, it becomes clear that under the imposition of the charge-neutrality condition, the occurrence of EDL can only be realized by charge separation inside the computational domain. In particular, the surface charge density must occur inside the fluid-solid interface, through some form of charge separation mechanism that can break local charge neutrality. This point and its implications are detailed in the next section.

\section{SURFACE POTENTIAL TRAP AND THE CHEMICAL POTENTIAL}

\section{A. Surface potential trap model}

Interfacial boundary conditions should ideally be independent of the bulk characteristics, such as $n^{\infty}$. However, it is well known that in the EO effect, experimental evidence indicates that the $\zeta$ potential varies as a function of $n^{\infty}$ $[105,106]$, e.g., by adding salt. Titration experiments have shown that the surface charge should be dependent on the $\mathrm{pH}$ and ionic strength [47]. Such correlation between $n^{\infty}$ and $\zeta$ implies that the interfacial charge density should be a derived quantity, i.e., the outcome of the calculation rather than an input.

As an alternative that can accommodate the variation of the $\zeta$ potential and the surface charge density, as well as provide a simple mechanism of generating EDL within the framework of the overall charge neutrality, we propose a (charge-neutral) surface potential trap model at the fluidsolid interface to serve as the ingredient for generating a surface charge layer $\sigma$. To motivate this model, let us consider the silica-water interface as an example. The conventional picture, as well as the reactive molecular dynamics simulation results [87], show that there exist dangling bonds, $\mathrm{Si}$ and $\mathrm{Si}-\mathrm{O}$, on a freshly cut silica surface. When the silica surface comes into contact with water, one neutral water molecule can dissociate into an $\mathrm{OH}^{-}$and an $\mathrm{H}^{+}$, which combine, respectively, with $\mathrm{Si}$ and $\mathrm{Si}-\mathrm{O}$ to form two silanol $(\mathrm{SiOH})$ groups. The silanol group is understood 
to be unstable in an aqueous environment and can easily lose or gain a proton, but this proton must stay in the neighborhood of the interface, owing to the electrostatic interaction. In this manner, an electrical double layer is established. It is clear that the interfacial ions generated in this fashion, denoted by $\sigma$, are in addition to $n^{\infty}$, which is the result of the law of mass action $\left(\mathrm{H}_{2} \mathrm{O} \rightleftharpoons \mathrm{OH}^{-}+\mathrm{H}^{+}\right)$of the water molecules.

It should be noted that there has been a tremendous amount of work in the area of the silica-water interface [107-111]. It is the intention of this work to address only the basic issue of interfacial charge separation with a simple model. More layers of complexities may be added to the basic theory in the future.

In order to simulate the process of surface charge layer generation, a (charge-neutral) surface potential trap is proposed, illustrated in Fig. 2(a). The surface potential trap should be specific to the type of ions. In the context of the silanol group example given above, the depth of the trap is indicative of the (free) energy relevant to the charge dissociation process. In other words, our model attributes a constant (free) energy cost to each ion pair generated. This charge-neutral surface potential trap can be either positive or negative, depending on the physical properties of the fluid-solid interface. In the case of the silica-water interface, the surface potential is positive so as to trap negative ions. For a fixed surface potential trap, it can be easily shown that the surface change density $\sigma$ would vary as a function of $a$ [Fig. 2(b)] and pH values [Fig. 4(a)] because $\sigma$ is now located inside the computational domain and must therefore be in active equilibrium with the bulk. This fact accounts for the basic distinction of the present model from the PB (CP) and GC (CC) models; it is a step closer to the observed reality. The use of the surface potential trap model, in conjunction with the PNP equations, also enables a consistent treatment of the time variation in the EO effect since in such a situation the local charge density can be time varying, and a fixed surface potential trap can accommodate the associated variation in the adsorbed surface charge density.

To implement the surface potential trap as part of the "interfacial condition," let us consider again a cylindrical channel with radius $a$. The surface potential trap function $f(r)$ has two parameters, the height of the trap, $\gamma$, and its width $\Delta$ :

$$
\begin{gathered}
f(r)=\frac{\gamma}{2}\left(1+\cos \frac{\pi(r-a)}{\Delta}\right), \quad \text { for } a-\Delta \leq r \leq a, \\
f(r)=0 \quad \text { for } 0 \leq r \leq a-\Delta .
\end{gathered}
$$

In what follows, we first consider the potential trap that is specific to the adsorption and desorption of $\mathrm{H}^{+}$from silanol groups. We shall set $\Delta$ to be the length of a dangling bond,
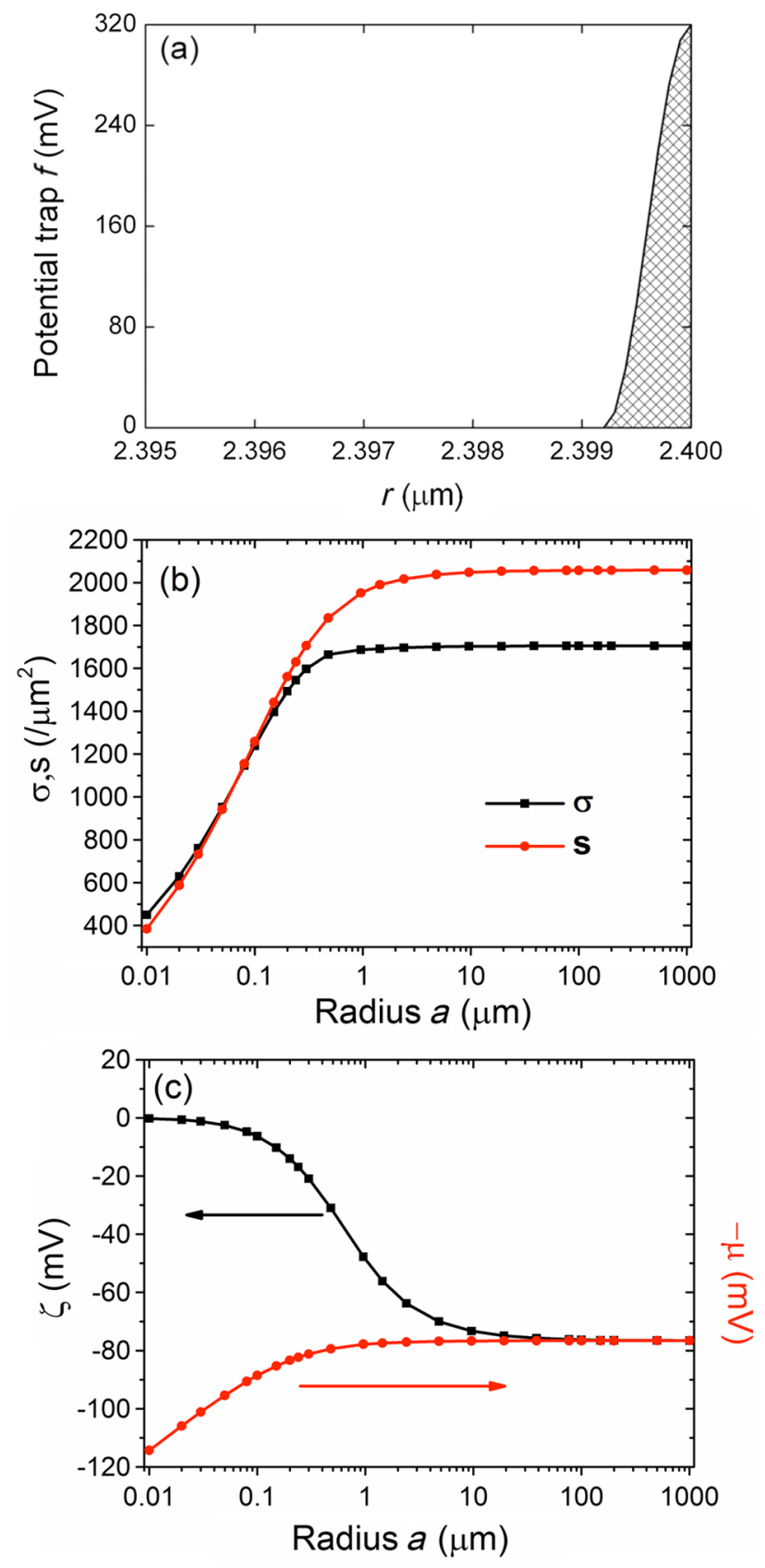

FIG. 2. Illustration of the interfacial potential trap and its consequences, with comparisons to the other models. (a) The functional form of the surface potential, given by Eq. (7). Here, the fluid-solid interface is at $r=2.4 \mu \mathrm{m}$. (b) The self-consistently determined surface charge density $\sigma$ as defined by Eq. (11), plotted as a function of $a$ (red symbols and curve). The black symbols and the fitting curve are for $s$, defined as the density of the negative ions integrated over the width of the surface potential trap shown in (a). It is seen that $s>\sigma$ because part of $s$ is captured from the bulk. (c) Negative of the chemical potential, $-\mu$ (right scale, red symbols and fitting curve), determined self-consistently with $\sigma$ through Eqs. (10b) and (11), plotted as a function $a$. The black symbols and fitting curve denote the zeta potential, $\zeta$ (left scale), as defined by Eq. (15). It is seen that the two quantities agree closely for $a>10 \mu \mathrm{m}$ but deviate from each other below that. 

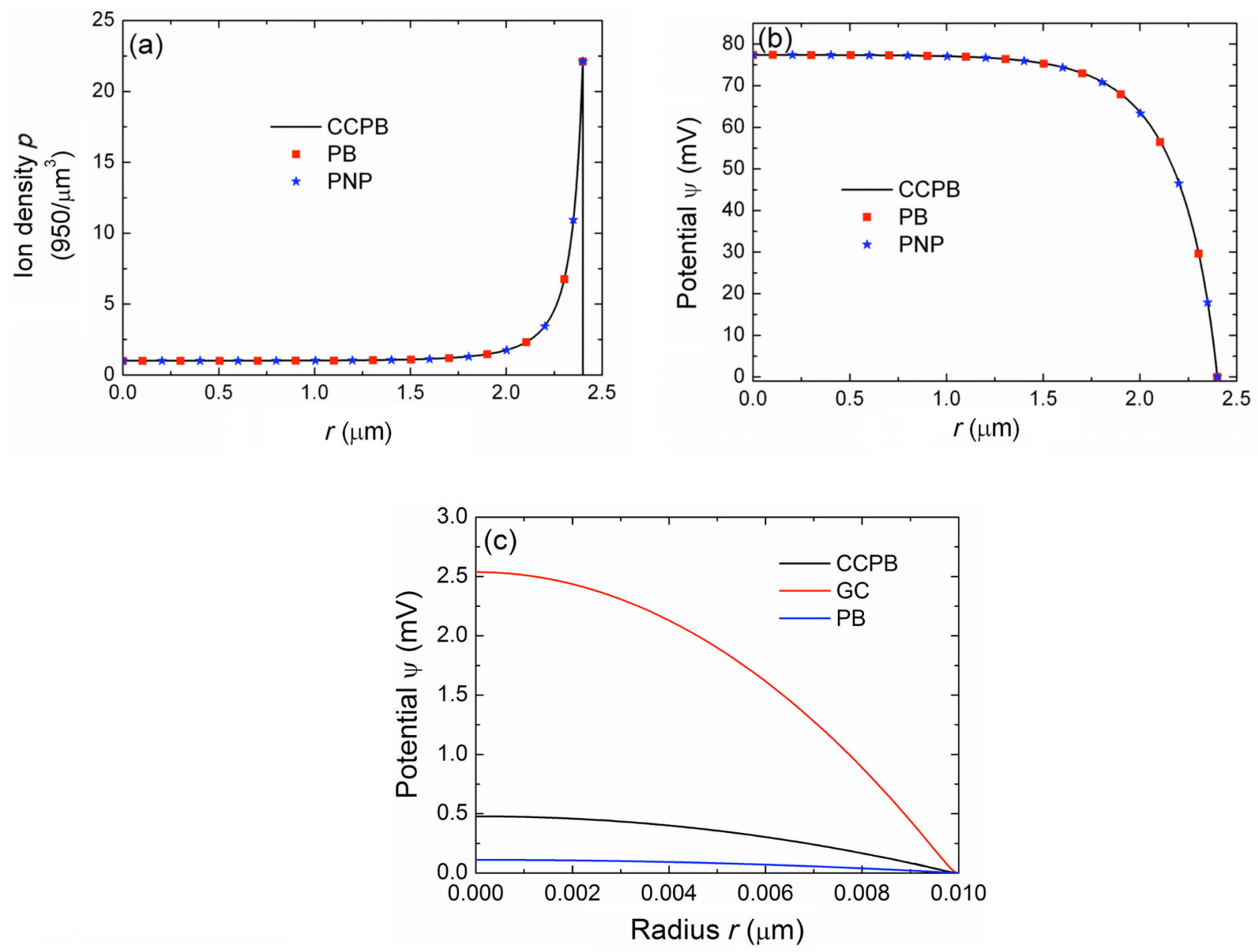

FIG. 3. (a) The distribution of the positive ions for $a=2.4 \mu \mathrm{m}$. Almost exact agreement is seen between the predictions of the PB and CCPB equations. The static limit of the PNP equations is also plotted, which (not surprisingly) agrees exactly with the CCPB. Not shown are the predictions of the Gouy-Chapman model (CC), which is exactly the same as that for the PB (CP) equation, with the Neumann boundary condition that corresponds to a surface charge density of $s=2058 / \mu \mathrm{m}^{2}$. At $a=2.4 \mu \mathrm{m}$, the actual value of $s=2016.8 / \mu \mathrm{m}^{2}$. (b) The potential profile for the Debye screening layer for $a=2.4 \mu \mathrm{m}$. Again, almost exact agreement is seen between the three cases. (c) The potential profile for the PB (blue line) and CCPB (black line) equations, plus the Gouy-Chapman (red line) model when $a=10 \mathrm{~nm}$. In contrast to (a) and (b), significant differences are seen for the predicted potential profiles. For the Gouy-Chapman model (CC), we use the same value of the surface charge density as that for the larger $a$, i.e., $2058 / \mu \mathrm{m}^{2}$. For the PB (CP) case, the value of $\zeta=-76.55 \mathrm{mV}$ has been used as the boundary condition. In the CCPB case, the use of either $-\mu=-114.3 \mathrm{mV}$ or $\sigma=s=450 / \mu \mathrm{m}^{2}$ would yield the result shown by the black curve in (c). For ease of comparison, in all cases the potential value at the boundary is shifted to zero.

$8 \AA$ A. It turns out that by fitting $\gamma$ so as to reproduce the $\zeta$ potential of water with $\mathrm{pH} 8.2\left(n_{\mathrm{OH}^{-}}^{\infty}=950 / \mu \mathrm{m}^{3}\right.$ and $\zeta=-76.55 \mathrm{mV}$, with $\mathrm{pH}$ values adjusted by the addition of $\mathrm{NaOH}$ ), we obtain $\gamma=320 \mathrm{mV}$. This corresponds roughly to the energy scale of a hydrogen bond; i.e., the potential trap is capable of breaking a hydrogen bond so as to release a proton. We note that the functional form of $f(r)$ cannot be arbitrary. In other words, the potential trap must be related, through the Poisson equation, to a fixed charge density $\rho_{c}$. Since our potential trap should be charge neutral, the volume integral of $\rho_{c}$ is constrained to be zero. Particularly, since $f(r)$ must satisfy the Poisson equation

$$
\frac{1}{r} \frac{\partial}{\partial r}\left[r \frac{\partial f(r)}{\partial r}\right]=-\frac{\rho_{c}}{\varepsilon},
$$

the integration of $\rho_{c}$ over the domain $a-\Delta \leq r \leq a$ should yield zero; i.e., the potential trap does not bring any external net charges into the system. It is easy to demonstrate that the form of $f$ given by Eq. (7) satisfies this constraint.

The potential distribution in the presence of the surface potential trap can be obtained by setting $\psi(r)=\varphi(r)-f(r)$, where $\psi(r)$ denotes the potential associated with the ions. From Eq. (7b), it is clear that $\psi=\varphi$ for $r<a-\Delta$. The potential function $\psi(r)$ satisfies the following CCPB equation: 


$$
\begin{aligned}
\frac{1}{r} \frac{\partial}{\partial r}\left(r \frac{\partial \psi}{\partial r}\right)= & \frac{e a^{2} n^{o}}{2 \varepsilon}\left\{\frac{\exp \left[e(\psi+f) /\left(k_{B} T\right)\right]}{\int_{0}^{a} \exp \left[e(\psi+f) /\left(k_{B} T\right)\right] r d r}\right. \\
& \left.-\frac{\exp \left[-e(\psi+f) /\left(k_{B} T\right)\right]}{\int_{0}^{a} \exp \left[-e(\psi+f) /\left(k_{B} T\right)\right] r d r}\right\} .
\end{aligned}
$$

In Fig. 2(a), we illustrate a particular surface potential trap with a height of $320 \mathrm{mV}$ and a width of $8 \AA$, situated at the fluid-solid interface for a cylindrical channel with $a=2.4 \mu \mathrm{m}$. It is noted that $f(r)=0$ at $r \leq a-\Delta$ Ultimately, this sets the reference potential value for the CCPB in this work. Equation (9) can be solved by using the finite-element COMSOL multiphysics package with the boundary conditions, as noted previously. The Neumann condition $\partial \psi / \partial r=0$ is used at the center of the cylindrical channel, and the Dirichlet condition $\psi(a)=$ 0 is imposed at the fluid-solid interface. It is shown below that the solution to the CCPB equation agrees exactly with the static limit of the PNP equations [Figs. 3(a) and 3(b)].

\section{B. Reformulation of the CCPB with the definition and self-consistent determination of chemical potential and surface charge density}

The addition of a surface potential trap breaks the symmetry between $p$ and $n$ near the fluid-solid interface. In order to better track the charge conservation as well as the surface charge density thus generated, we reformulate CCPB by noting that Eq. (9) can be written alternatively as

$$
\begin{aligned}
\frac{1}{r} \frac{\partial}{\partial r}\left(r \frac{\partial \psi}{\partial r}\right)= & \frac{e n^{\infty}}{\varepsilon}\left\{\exp \left[e(\psi-\mu+f) / k_{B} T\right]\right. \\
& \left.-\exp \left[-e(\psi-\mu+f) / k_{B} T\right]\right\},
\end{aligned}
$$

where the chemical potential $\mu$ is defined by the chargeneutrality condition

$$
\mu=\frac{k_{B} T}{2 e} \ln \left\{\frac{\left.\int_{0}^{a} \exp \left[e(\psi+f) / k_{B} T\right)\right] r d r}{\int_{0}^{a} \exp \left[-e(\psi+f) / k_{B} T\right] r d r}\right\} .
$$

In Eq. (10a), it should be noted that we use $n^{\infty}$ on the righthand side, instead of $n^{o}$ as in Eq. (9). The electrical potential is now referenced to the chemical potential. However, it is easy to see that when Eqs. (10a) and (10b) are considered together, the property of reference potential independence is still preserved. In Appendix B, we show that Eq. (10b) can be derived from the free energy, with $\mu$ serving as the Lagrange multiplier for the chargeneutrality constraint.

The additional condition - that the integral of each term on the right-hand side of Eq. (10a) must be identical to the integral of the same term in Eq. (9) —yields a dimensionless constant $\kappa$ :

$$
\begin{aligned}
& \frac{2}{a^{2}} \int_{0}^{a} \exp \left[e(\psi-\mu+f) / k_{B} T\right] r d r= \\
& \kappa=\frac{2}{a^{2}} \int_{0}^{a} \exp \left[-e(\psi-\mu+f) / k_{B} T\right] r d r,
\end{aligned}
$$

with the constraint that $n^{\infty} \kappa=n^{o}$. Since the surface potential trap inevitably generates a surface charge layer, there is a relationship between $n^{o}, n^{\infty}$, and $\sigma$ :

$$
n^{o}=n^{\infty}+2 \frac{\sigma}{a}
$$

It follows that $\sigma=n^{\infty}(\kappa-1) a / 2$. But the value of $\kappa$ depends on $\mu$, which requires $\sigma$ (as part of $n^{o}$ ) as one of the input parameters. Hence, there is a consistency condition that may be expressed succinctly as $\sigma=n^{\infty} a\{\kappa[\mu(\sigma)]-1\} / 2$.

\section{Generalization to the case of surface nonspecific salt ion addition}

Salt addition is commonly used to vary the ion concentration. From the retention times in ion-exchange chromatography, a series of binding affinities can be ordered as $\mathrm{Cs}+>\mathrm{K}+>\mathrm{Na}+>\mathrm{Li}+[112,113]$. This is easy to understand from the viewpoint that ions with an increasing hydration shell are more prone to stay in water. For simplicity, we assume that the surface-specific salt ions can be treated just as the water ions. For example, for $\mathrm{NaOH}$, the $\mathrm{OH}^{-}$ions are of course the same as that from water, and for the $\mathrm{Na}^{+}$ions, they can be treated on the same basis as $\mathrm{H}^{+}$since the positive ions are all repulsed by the surface potential trap.

For those salt ions which do not interact with surface silanol groups, i.e., surface nonspecific salt ions such as $\mathrm{Na}^{+} / \mathrm{Cl}^{-}$, the same theoretical framework can be generalized to the following form, based on the observation that while the salt ions would electrically interact with all the other ions, they do not feel the effect of a surface potential trap. Hence, the CCPB equation (9) can be written as

$$
\begin{aligned}
\frac{1}{r} \frac{\partial}{\partial r}\left(r \frac{\partial \psi}{\partial r}\right)= & \frac{e}{\varepsilon}\left\{n_{\mathrm{OH}^{-}}^{\infty} \exp \left[e(\psi-\mu+f) / k_{B} T\right]\right. \\
& +n_{\mathrm{Cl}^{-}}^{\infty} \exp \left[e(\psi-\mu) / k_{B} T\right] \\
& -n_{H^{+}}^{\infty} \exp \left[-e(\psi-\mu+f) / k_{B} T\right] \\
& \left.-n_{\mathrm{Na}^{+}}^{\infty} \exp \left[-e(\psi-\mu) / k_{B} T\right]\right\},
\end{aligned}
$$

where the chemical potential $\mu$ is determined by the charge-neutrality condition 


$$
\mu=\frac{k_{B} T}{2 e} \ln \left\{\frac{\int_{0}^{a}\left\{n_{\mathrm{OH}^{-}}^{\infty} \exp \left[e(\psi+f) / k_{B} T\right]+n_{\mathrm{Cl}^{-}}^{\infty} \exp \left[e \psi / k_{B} T\right]\right\} r d r}{\int_{0}^{a}\left\{n_{H^{+}}^{\infty} \exp \left[-e(\psi+f) / k_{B} T\right]+n_{\mathrm{Na}^{+}}^{\infty} \exp \left[-e \psi / k_{B} T\right]\right\} r d r}\right\} .
$$

We note that the charge-neutrality relation between the ion densities of the four species at infinity is given by

$$
n_{\mathrm{OH}^{-}}^{\infty}+n_{\mathrm{Cl}^{-}}^{\infty}=n_{\mathrm{Na}^{+}}^{\infty}+n_{H^{+}}^{\infty}=n^{\infty} \text {. }
$$

The additional condition - that the integral of the negative or positive ion density on the right-hand side of Eq. (12a) must be identical to the integral of the same term in Eq. (9)-yields the following equation:

$$
\begin{aligned}
& \frac{2}{a^{2}} \int_{0}^{a}\left\{n_{\mathrm{OH}^{-}}^{\infty} \exp \left[e(\psi-\mu+f) / k_{B} T\right]+n_{\mathrm{Cl}^{-}}^{\infty} \exp \left[e(\psi-\mu) / k_{B} T\right]\right\} r d r=n^{o} \\
& \quad=\frac{2}{a^{2}} \int_{0}^{a}\left\{n_{H^{+}}^{\infty} \exp \left[-e(\psi-\mu+f) / k_{B} T\right]+n_{\mathrm{Na}^{+}}^{\infty} \exp \left[-e(\psi-\mu) / k_{B} T\right]\right\} r d r .
\end{aligned}
$$

It should be noted that in the above generalization, we have used the presence or absence of a single surface potential trap $f$ in the distribution function of the various ion specifies, in order to differentiate their affinities to the solid surface. An obvious direction to further generalize the model is to use different surface potential traps, e.g., one for each ion species $\left(f_{H^{+}}\right.$for protons, $f_{\mathrm{Na}^{+}}$for sodium ions, and $f_{\mathrm{Cl}^{-}}$for chlorine ions), to characterize their interfacial affinities. However, it is the purpose of the present work to give the simplest version and to demonstrate the model's effectiveness even in its minimal edition.

In actual calculations, we first solve Eq. (12a) for $\psi$ (with given $n_{\mathrm{OH}^{-}}^{\infty}, n_{\mathrm{Cl}^{-}}^{\infty}, n_{\mathrm{Na}^{+}}^{\infty}, n_{H^{+}}^{\infty}$ and a guess for $\sigma$ ), which is subsequently used in Eq. (12b) to obtain $\mu$. Then, Eqs. (12d) and (11) are used to check the consistency between the initial value $\sigma$ and that deduced from $n^{o}$. If it is found to be inconsistent, then a new value of $\sigma$ is determined from the consistency relation, and iterations are carried out until consistency is achieved. In this manner, one obtains $\sigma$ and $\mu$ simultaneously from the input $\mathrm{pH}$ and $\mathrm{pC}$ values. The interpretation is that if we hold $n^{\infty}$ to be a given constant, then the surface potential trap needs to generate a surface charge density $\sigma$ (and its counterions) in order to retain consistency between the input $n^{o}$ to Eq. (9), and the resulting ion density allocation by the CCPB (through its solution $\psi$ ) to the potential trap and the bulk ion density. The latter must be held at $n^{\infty}$. In Fig. 2(b), we show the variation of $\sigma$ as a function of $a$ with $n^{\infty}=$ $950 / \mu \mathrm{m}^{3}$ as the input value ( $\mathrm{pH}=8.2$, adjusted by $\left.\mathrm{NaOH}\right)$. It is seen that $\sigma$ is a constant for large $a$, as it should be, but decreases significantly when the Debye layers from the two opposite surfaces overlap. At $a=10 \mathrm{~nm}$, we have $\sigma=450 / \mu \mathrm{m}^{2}$, which is only a quarter of the value at the $a \rightarrow \infty$ limit. Also plotted, in symbols, is the surface ion density, $s$, obtained by directly integrating the captured negative ions in the surface potential trap region (the amount of positive ions in the trap is less than $1 \%$ ). Here, $s$ represents the charge density that should exactly cancel the net charge in the diffuse Debye screening layer.
It is the total charge in the Stern layer. A difference between $s$ and $\sigma$ is seen, owing to the fact that $s$ contains some ions captured from the bulk, whereas $\sigma$ represents the surfacespecific adsorbed ions generated by the break-up of neutral molecules by the surface potential trap. However, when the radius decreases below $\lambda_{D}, s$ is seen to approach $\sigma$.

The surface charge density variation seen in Fig. 2(b), usually denoted as the charge regulation phenomenon, is indicative of the fact that the surface charge layer density is subject to variation when the interfacial environment changes. Here, the surface charge density is coupled to the bulk as a consequence of being within the computational domain [see also Fig. 4(a)]; hence, its variation is both necessary and guaranteed to conform with the principle of free energy minimization. Traditionally, the "correct" variation as implied by the experimental measurements is sometimes obtained by interpolating between the $\mathrm{CC}$ boundary condition and the $\mathrm{CP}$ boundary condition, with an interpolation parameter that is fitted to the experimental data. Here, the desired behavior emerges naturally, with no additional parameters. It is shown below that such surface charge density variation is crucial to understanding the experimental data obtained at the nanometer scale channel width.

In Fig. 2(c), we show the associated variation of $-\mu$ plotted as a function of $a$, where the minus sign in front of $\mu$ is installed in anticipation of its comparison with the zeta potential.

Why does $\sigma$ decrease with decreasing $a$ ? Basically, it is a matter of energetics. Since the interfacial charge separation is associated with a given energy, with decreasing $a$ (to below $\lambda_{D}$ ), the repulsive interaction between the positive ions in the screening layer would increase with increasing $n^{o} \sim 2 \sigma / a$. The overall free energy minimization is what dictates the behavior seen in Fig. 2(b).

\section{Reduction to the PB equation form}

By noting that $f(r)=0$ for $r \leq a-\Delta$, Eq. (12a) may be written in the form 

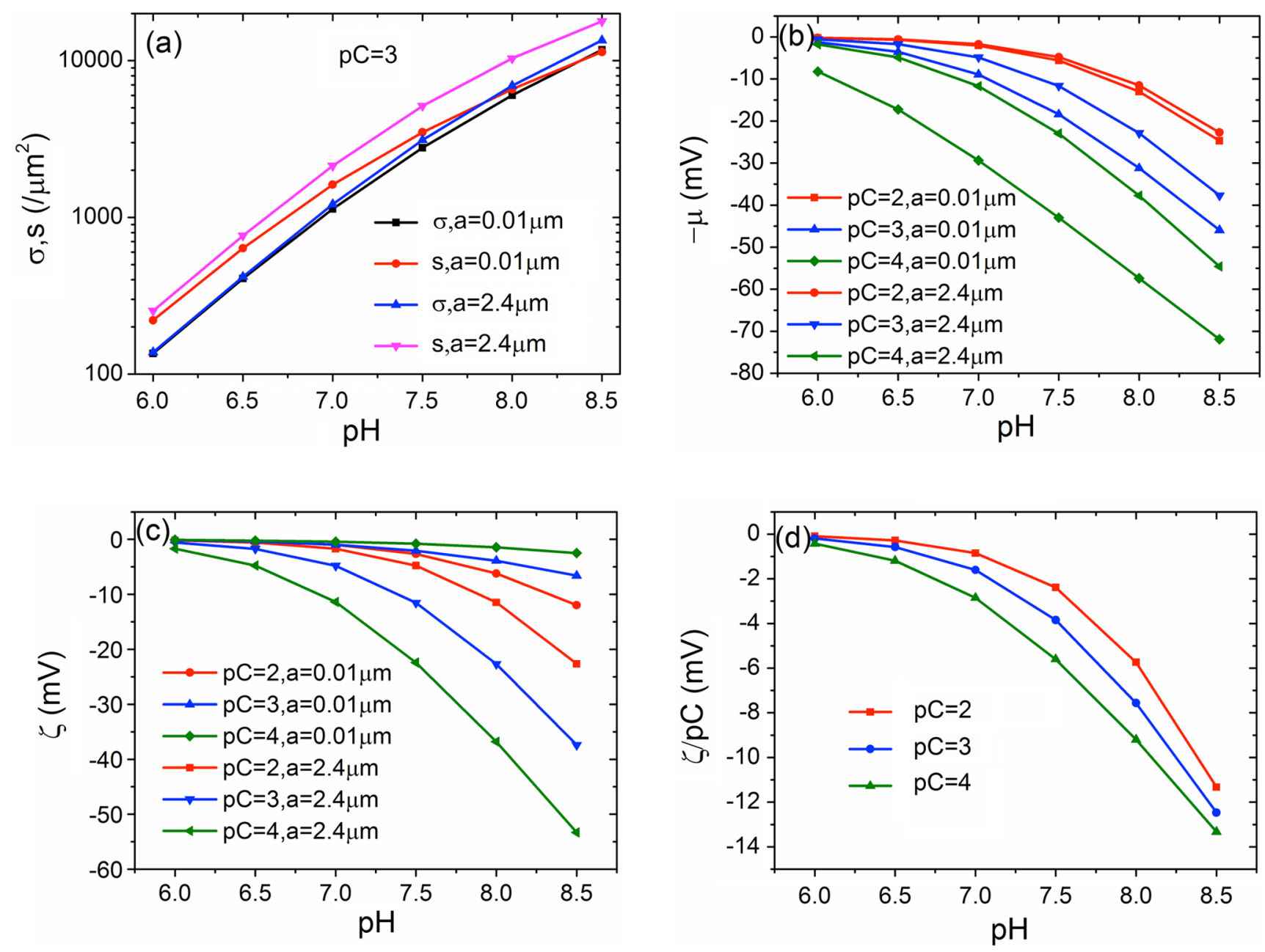

FIG. 4. Correlation of the modeling parameters with the channel radius and $\mathrm{pH}$ values. The magnitude of the surface potential is set at $\gamma=320 \mathrm{mV}$. (a) Correlation between the surface charge density and $\mathrm{pH}$ values, for two cases of $a=2.4$ and $0.01 \mu \mathrm{m}$ under $\mathrm{pC}=3$. In both cases, the value of $s$ tracks $\sigma$. (b) Chemical potential $\mu$ plotted as a function of $\mathrm{pH}$ values, for a set of different $a$ 's and $\mathrm{pC}$ values ranging from 2 to 4 . (c) $\zeta$ potential plotted as a function of $\mathrm{pH}$ values, for a set of different $a$ 's and $\mathrm{pC}$ values ranging from 2 to 4 . (d) $\zeta / \mathrm{pC}$ plotted as a function of $\mathrm{pH}$ values for $a=2.4 \mu \mathrm{m}$ and $\mathrm{pC}$ values ranging from 2 to 4 . It is seen that the $|\zeta|$ varies linearly with $\mathrm{pH}$ values for $a>\lambda_{D}$. This conforms with the experimentally observed behavior. It is also seen that $|\zeta|$ decreases with channel radius $a$ but increases with increasing $\mathrm{pH}$ values. Here the $\zeta$ potential is consistently calculated from Eq. (15).

$$
\begin{aligned}
\frac{1}{r} \frac{\partial}{\partial r}\left(r \frac{\partial \psi}{\partial r}\right)= & \frac{e n^{\infty}}{\varepsilon}\left\{\exp \left[e(\psi-\mu) / k_{B} T\right]\right. \\
& \left.-\exp \left[-e(\psi-\mu) / k_{B} T\right]\right\}
\end{aligned}
$$

within the reduced domain. Simple manipulation leads to the form of the $\mathrm{PB}$ equation:

$$
\frac{1}{r} \frac{\partial}{\partial r}\left(r \frac{\partial \bar{\psi}^{(\mathrm{PB})}}{\partial r}\right)=\frac{1}{\lambda_{D}^{2}} \sinh \left(\bar{\psi}^{(\mathrm{PB})}\right) .
$$

Here, $\bar{\psi}^{(\mathrm{PB})}=e(\psi-\mu) / k_{B} T$, with $\psi^{(\mathrm{PB})}=\psi-\mu$. The boundary condition, applied at $r=a-\Delta$, should be $\psi^{(\mathrm{PB})}=-\mu$ because we have set $\psi(a)=0$, and therefore $\psi(a-\Delta) \rightarrow 0$ as $\Delta \rightarrow 0$ (in actual calculations, the difference from zero is at most a fraction of one $\mathrm{mV}$, which is noted to be of the same magnitude as the traditional potential difference between the Stern layer and surface layer). It follows that in our form of the PB equation, $-\mu$ plays the role of the traditional $\zeta$ potential. However, distinct from the traditional $\mathrm{PB}$ equation in which the $\zeta$ potential is treated as a constant, here $-\mu$ can vary with $a$ and/or $n^{\infty}$. Since the use of Eq. (14) with the accompanying $-\mu$ boundary condition leads to exactly the same predictions as the CCPB equation, it is fair to say that the consideration of the charge-neutrality constraint has led to a redefinition of the boundary condition for the PB equation, and the actual difference from the traditional approach becomes important only at the scale of nanometers to a few $\mu \mathrm{m}$. We note in passing that the above derivation of the PB equation also resolves the question, raised previously, regarding the correct boundary condition when $a<\lambda_{D}$. 


\section{E. Generalized definition of the $\zeta$ potential}

In contrast to the chemical potential that is defined on the basis of the charge-neutrality constraint, the $\zeta$ potential should be the basis of the EK effects that arises from charge separation and its associated potential inhomogeneities. For a cylindrical channel with radius $a$, it is shown in Appendix A that when the PB equation is coupled with the NS equation, with the traditional approximations, both the EO fluid flow and the related Onsager coefficient are proportional to the integral of the volume-averaged potential variation:

$$
\zeta=\frac{2}{a^{2}} \int_{0}^{a}\left[(-\mu)-\psi^{(\mathrm{PB})}\right] r d r=-\frac{2}{a^{2}} \int_{0}^{a} \psi(r) r d r,
$$

where $\psi$ denotes the solution to the CCPB equation. In Eq. (15), we have extended our integration to $r=a$ because the integrand is essentially zero from $a-\Delta$ to $a$. In Fig. 2(c), the calculated $\zeta$ potential is plotted as a function of $a$. It is seen that $\zeta \cong-\mu$ above $10 \mu \mathrm{m}$, but the two deviate from each other below that.

From Eq. (15), it is easy to see that in the $a \rightarrow \infty$ limit, we must have $\zeta=-\mu$ because in a large channel, except close to the boundary where there is charge separation, most of the channel must be charge neutral. Hence, from Eq. (10a) [or (12a)], we should have $\psi=\mu$ in the bulk, and the volume average of $\psi$ must equal $\mu$ since the interfacial contribution is negligible in this case. The potential variation in this case is just $-\mu$ (from $\psi=\mu$ in the bulk to $\psi=0$ at the interface). It should be noted that $\psi^{(\mathrm{PB})}=0$ in the bulk, as expected.

\section{F. Comparison and contrast with the traditional picture}

It is satisfying that the physical picture of GouyChapman (CC) can be reproduced within the computational domain, under the charge-neutrality constraint. The place where the PB equation's potential boundary condition (CP) must be applied, i.e., at $r=a-\Delta$, is noted to coincide precisely with that of the traditional theory. In distinction to the traditional PB model, here $-\mu$ is a function of $a$ and $n^{\infty}$; i.e., the boundary value is a derived quantity rather than a constant input. This is because in the present case, the PB equation form is defined only within $r \leq a-\Delta$; therefore, its boundary value must represent all the relevant information within the entire computational domain, reduced down to a single number. The clarification of the meaning of the traditional zeta potential as the chemical potential also shows that the use of the $\mathrm{CP}$ boundary condition is only valid at the large channel limit. But even that is suspect since the chemical potential can vary with salt addition, while the usual notion of a boundary condition should be particular only to interfacial characteristics.

While the surface charge density is determined by the boundary condition in the PB model [Eq. (4)], in the present case the boundary value $(-\mu)$ and $\sigma$ are both determined simultaneously by the surface potential trap and $n^{\infty}$. In contrast to the traditional theory, here $\sigma$ is situated within the computational domain as emphasized previously, and therefore, it is subject to variation in conjunction with the bulk input parameters.

The charge regulation phenomenon, denoting the fact that the surface charge density can vary as a function of separation and local chemical environment, is seen to emerge naturally in our model. Taking the silica-water interface for example $[85,86]$, previous treatments take advantage of the experimentally supported " $1-\mathrm{pK}$ basic Stern model" [114,115], which describes the various reactions that can occur at the silica-water interface. They are sensitive to the chemical nature of the interface and effective at separations larger than the Debye length. However, in our surface potential trap model, the surface reaction is depicted by the free energy cost for surface-active ions' desorption or adsorption. To a large extent, our model captures the essential physics underlying these complex reactions.

The present model differs from the traditional picture when the Debye layers of two charged surfaces overlap. In this situation, neither the diffuse layer charge nor the diffuse layer potential can remain constant, and both quantities vary with separation as a result of an interplay between the diffuse layer and the surface. As stated previously, when separation decreases, the dissociation reactions occuring at the surface are suppressed because of the minization of overall free energy.

It is well known that the charge layer adsorbed at the fluidsolid interface, e.g., in the Gouy-Chapman model, is regarded as the immobile "Stern layer." In our model, while movement normal to the interface is naturally limited by the surface potential trap, movement parallel to the interface is not artificially constrained. We show below that when this model is coupled with the NS equation, the adsorbed charges will have no movement parallel to the interface, owing to the no-slip boundary condition. Thus, the adsorbed (trapped) ions in this case can correspond accurately to the Stern layer. However, since we have not artificially fixed the adsorbed charges at the interface, the movement parallel to the interface can be enhanced by the slip hydrodynamic boundary condition, e.g., the Navier boundary condition. While the slip boundary condition might not be common to many of the physical systems, this added degree of freedom nevertheless makes the present model attractive for simulating a more diverse array of problems.

\section{COMPARISON WITH ALTERNATIVE MODELS AND EXPERIMENTS}

\section{A. Comparison between the predictions of the PB, $\mathrm{CCPB}$, and PNP equations}

By using the COMSOL multiphysics finite element package to numerically evaluate the surface potential trap model with the input parameters $a$ and $n^{\infty}(\Delta$ is set at $8 \AA$ ), 
the potential profile of the CCPB can be evaluated. We show in Figs. 3(a) and 3(b) that when $a$ is large, the positive ion density and the potential profile show almost exact agreement between the solutions of the $\mathrm{PB}$ equation [with $-\mu(a \rightarrow \infty)$ as the boundary condition], the CCPB equation, and the static limit of the PNP equations for $a=2.4 \mu \mathrm{m}$. If we use a Neumann boundary condition that corresponds to a surface charge density of $2058 / \mu \mathrm{m}^{2}$, i.e., the Gouy-Chapman model (CC), the prediction is also almost identical to that of the PB equation. The excellent agreement between all the different models holds down to $a \cong \lambda_{D}$, or about $300 \mathrm{~nm}$. However, differences emerge below that scale. In Fig. 3(c), it is shown that when $a=10 \mathrm{~nm}$, the predictions of the PB (CP) and CCPB equations differ quite significantly, especially in the value of the $\zeta$ potential, as can be inferred by the amount of potential variation [see Eq. (15)]. If we still use the same surface charge density of $2058 / \mu \mathrm{m}^{2}$ as in the Neumann boundary condition for the $a=10 \mathrm{~nm}$ case, then the GouyChapman model (CC) prediction is shown by the red line, which is above that of the $\mathrm{CCPB}$ or the $\mathrm{PB}(\mathrm{CP})$. This difference is due to the lower value of the trapped negative ion charge density in the CCPB case (or the static limit of the PNP equations), $450 / \mu \mathrm{m}^{2}$.

In regard to Fig. 3(b), it may be worthwhile to note the very simple physics involved. In other words, since the surface charge density and the diffuse screening layer essentially form a capacitor, there is necessarily a jump in potential from $\psi=0$ (boundary condition on $\psi$ ) at the fluid-solid interface to $\psi=\mu$ in the bulk (required by charge neutrality as noted previously). The transition distance is characterized by $\lambda_{D}$. This picture holds until the channel width $a$ becomes less than $\lambda_{D}$; then, the relevant physics can become more complicated, owing to the variation in the surface charge density and the incomplete screening such that the center of the channel is no longer charge neutral.

\section{B. Variation of surface charge, chemical, and zeta potentials with $\mathrm{pH}$ under different (surface nonspecific) salt concentrations}

Here, we focus on the CCPB equation and its various parameters. Inputs to the CCPB equation comprise $a, \mathrm{pH}$, $\mathrm{pC}$, and the surface potential trap height $\gamma$. By first determining $\sigma$ and $\mu$ consistently, the subsequent potential profile and the values of $\zeta$ can be easily obtained. In Figs. 4(a), 4(b), and 4(c), we show, respectively, the variations of $\sigma, \mu$, and $\zeta$ as a function of $\mathrm{pH}$ values. To test the empirical equation $\left(\zeta=a_{0}+a_{1} \cdot \mathrm{pC}\right.$, where $a_{0}$ and $a_{1}$ are functions of the $\mathrm{pH}$, temperature, substrate material, and counterion type), we normalize the zeta potential with $\mathrm{pC}$ values, as shown in Fig. 4(d). For the theory results, normalized zeta potentials display a nearly linear variation as a function of $\mathrm{pH}$ with a slope of $6.5 \mathrm{mV} / \mathrm{pH}$ (for $\mathrm{pH}>7$ ), comparable to the experimental value of
4-7 mV/pH [106]. Hence, our model can provide a rather good explanation of the measured zeta potential results, which have also been addressed by previous models with varying complexities [116,117]. In Fig. 4(a) (take $\mathrm{pC}=3$, for example), it is seen that the variation of $\sigma$ may be described by a surprisingly simple expression: $\ln \sigma \sim \ln n^{\infty}$ when $a=2.4 \mu \mathrm{m}$. However, this can change when $a=10 \mathrm{~nm}$. Figure 4(b) also shows that $\mu$ increases in magnitude with increasing $\mathrm{pH}$ values and decreasing $a$. But the $\zeta$ potential, shown in Fig. 4(c), has the opposite variation with $a$, owing to the finite width of the Debye screening layer, so that when $a \leq \lambda_{D}$, the potential
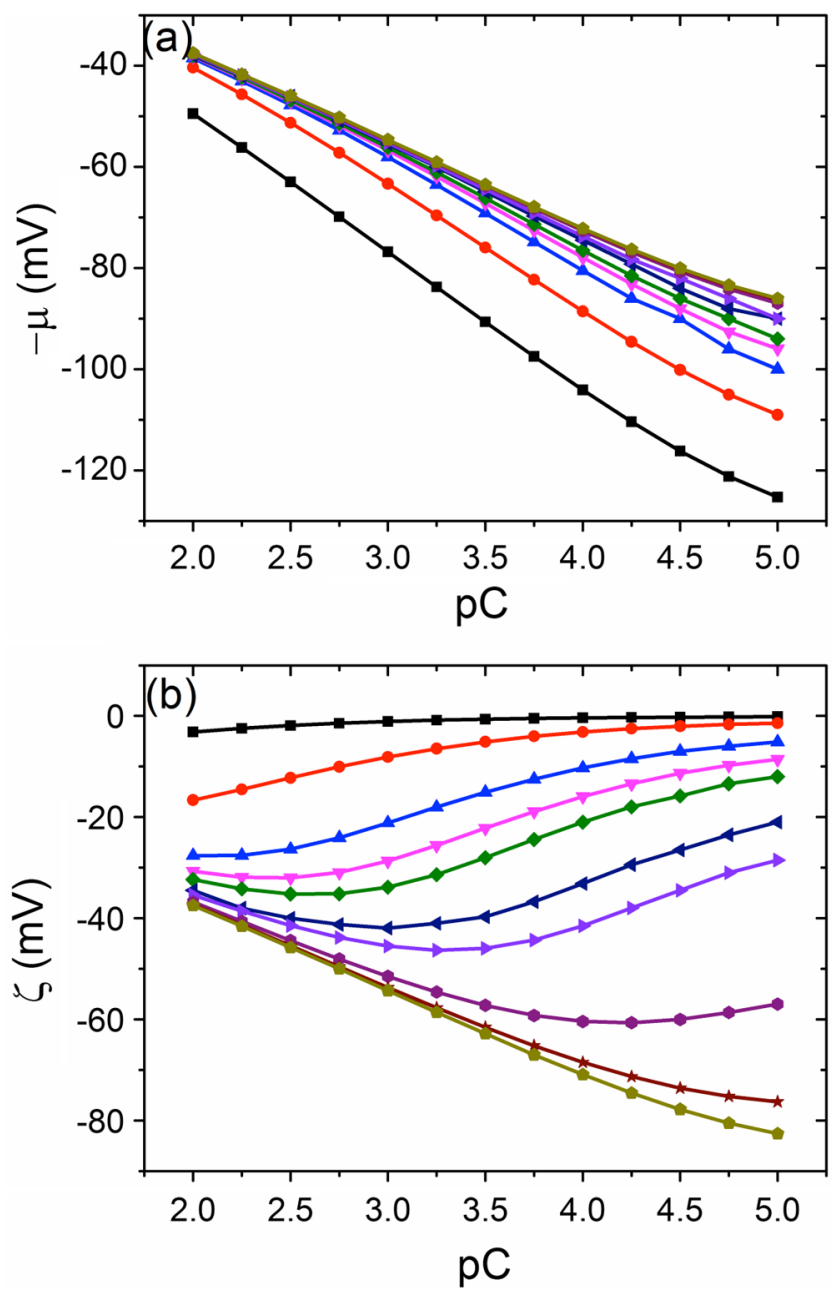

FIG. 5. (a) Chemical potential $-\mu$ plotted as a function of salt ion concentration $\mathrm{pC}$ under $\mathrm{pH} 9$ for a set of different radii $a$. (b) $\zeta$ potential plotted as a function of the negative logarithm of salt ion concentration, $\mathrm{pC}$, under $\mathrm{pH} 9$, for a set of different radii $a$. In (a), the different curves represent the effect of different channel radius. From top downward, the radius are 3, 1, 0.3, 0.1, 0.07, $0.04,0.03,0.02,0.008$, and $0.002 \mu \mathrm{m}$. The order is reversed in (b), with the largest radius curve at bottom. It should be noted that for a channel width of $40 \mathrm{~nm}$ to $300 \mathrm{~nm}$, the zeta potential displays a maximum in its absolute value, just as observed experimentally. 
difference between the fluid-solid interface and the center of the channel becomes smaller [118,119]. The EK effect diminishes as a result. Both $\zeta$ and $\mu$ can vary by a significant amount with a decreasing salt addition, owing to the less prominent screening effect at lower salt concentrations.

\section{Variation of surface charge, chemical, and zeta potentials with $\mathbf{p C}$}

As stated previously, we distinguish between two different types of salt. Salt with ions that can be surface-specific adsorbed can have a very different effect from those salts whose ions cannot interact with the surface potential trap, i.e., the non-surface-specific ions. Sodium hydroxide, $\mathrm{NaOH}$, is a salt whose $\mathrm{OH}^{-}$ions can surface-specific adsorb onto the silica-water interface and hence can be treated on the same basis as water ions in regard to the surface potential trap. Non-surface-specific ions are from those salts, e.g., $\mathrm{KCl}$ or $\mathrm{NaCl}$, whose ions cannot react with the interfacial dangling bonds; yet they contribute to the screening effect and can therefore decrease the Debye length. These two types of salt additions have to be modeled differently. While in the previous section we model the effect of $\mathrm{pH}$ values, in this section we illustrate the effect of the added salt (nonspecific type). It will be shown that they have the opposite effect with respect to the $\zeta$ and $\mu$.

It should be cautioned that by increasing the concentration of a surface-specific type of salt, the interface may eventually experience a saturation effect and charge reversion $[106,118,120]$, which are not contained in the present model. Hence, the modeled behavior may differ from the experimental behavior at the limit of very large ion density.

In Fig. 5, we plot the variation of $-\mu$ [Fig. 5(a)] and $\zeta$ [Fig. 5(b)] as a function of $\mathrm{pC}$ under $\mathrm{pH} 9$. In contrast to the traditional theory prediction that the magnitude of the zeta potential should display monotonic variation with decreasing salt concentration (but opposite in direction for the $\mathrm{CP}$ and CC boundary conditions), here, at channel width smaller than $0.3 \mu \mathrm{m}$, the zeta potential displays a peak in its absolute magnitude. Furthermore, as the channel width further decreases, the peak position moves towards a higher salt concentration regime (smaller values of $\mathrm{pC}$ ). This is precisely the behavior observed in streaming current measurements within silica nanochannels [78]. Since the streaming current is proportional to the zeta potential, the precisely similar behavior observed there lends great support to our simple theory. Besides, there is necessarily a straight-line section of the curve that exhibits a $(-\mu, \zeta) \sim$ $\ln C$ behavior at radius larger than the Debye length, which has been experimentally observed [106,118]. The slope of the straight-line section, around $\sim 18 \mathrm{mV}$ per one decade of variation in $\mathrm{C}$, is also on the same order as that measured experimentally, which is around $\sim 22.5 \mathrm{mV}$ per one decade of variation in $\mathrm{C}$ at $\mathrm{pH} 9$ [121]. However, we note that such behavior does not hold at very high nonspecific adsorption salt concentrations since the value of the potential jump must necessarily tend toward zero. This aspect also agrees with the experiment. Thus, our rather simple model, with no adjustable or added parameters, can naturally lead to predictions that are in very reasonable agreement with experimental observations.

\section{FORCE BETWEEN TWO CHARGED SURFACES: COMPARISON BETWEEN THEORY AND EXPERIMENT}

There have been numerous experiments measuring the force between two charged surfaces. In order to compare the prediction of our theory with the experimental data, we start by calculating the free energy of the whole system composed of two planar surfaces, at given separation. The pressure is then calculated by numerically differentiating the free energy per unit area with respect to the separation. Here, we write the free energy per unit area as

$$
F=\frac{k_{B} T}{A} \int_{\Omega} d x\left(n_{\mathrm{Na}^{+}} \ell n \frac{n_{\mathrm{Na}^{+}}}{n^{o}}+n_{H^{+}} \ell n \frac{n_{H^{+}}}{n^{o}}+n_{\mathrm{OH}^{-}} \ell n \frac{n_{\mathrm{OH}^{-}}}{n^{o}}+n_{\mathrm{Cl}^{-}} \ell n \frac{n_{\mathrm{Cl}^{-}}}{n^{o}}+\frac{\varepsilon}{2 k_{B} T}|\nabla \psi|^{2}+\frac{e f N}{k_{B} T}\right) .
$$

Here, $A$ is the total area, $\Omega$ denotes the relevant domain of integration, $d \boldsymbol{x}$ denotes volume integration, $\varepsilon$ denotes the dielectric constant, $N$ is the $\mathrm{H}^{+} / \mathrm{OH}^{-}$ion density in the potential trap, and $f$ denotes the surface potential trap function. Since the system is composed of four ion species, $\mathrm{H}^{+} / \mathrm{OH}^{-}$and $\mathrm{Na}^{+} / \mathrm{Cl}^{-}$, the free energy naturally comprises the mixing entropy as well as the electrostatic interaction energies.

It should be noted that, experimentally, the force is directly measured by the experimental apparatus, such as the AFM, between a flat surface and a spherical particle. After obtaining the force data, it is usually divided by an effective radius obtained from the commonly used Derjaguin approximation, taking account of the relevant geometry. Here, we calculate the pressure between two planar surfaces at every separation by differentiating the free energy per unit area, then sum them together after multiplying by the differential area element, in accordance with the varying separations as dictated by the geometry between a sphere and a flat plane. The resulting force is then divided by the experimentally used effective radius for comparison to data.

The free energy shown in Fig. 6 displays repulsion between two charged surfaces but attraction at a separation 


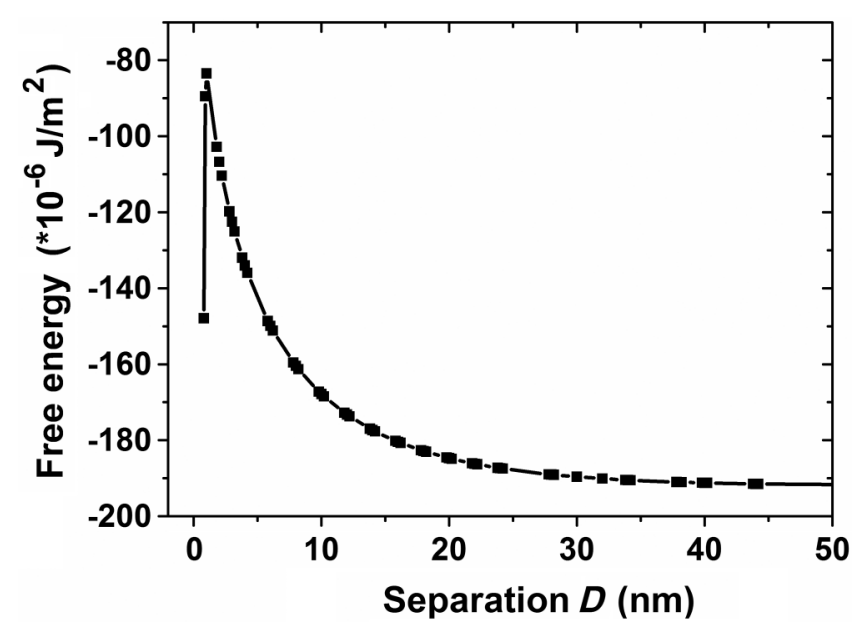

FIG. 6. The calculated free energy per unit area of silica surfaces immersed in 1-mM $\mathrm{NaNO}_{3}$ under $\mathrm{pH} 7$, plotted as a function of separation distance between two planar charged surfaces.

distance smaller than $1 \mathrm{~nm}$. This latter phenomenon was, in fact, observed experimentally [122]. At separation smaller than $2 \mathrm{~nm}$, a strong attraction was observed, the gradient of which can cause the surfaces to jump together. At larger separations where the force curves are charaterized by an exponentially decreasing replusive component, we see in Figs. 7 and 8 that there is indeed a reasonable agreement between the predictions of our model and the experiments. It should be remarked that such agreement has been obtained without adjusting the theory parameters, such as the potential trap depth (which should be fixed by the zeta-potential value in the large-channel limit).

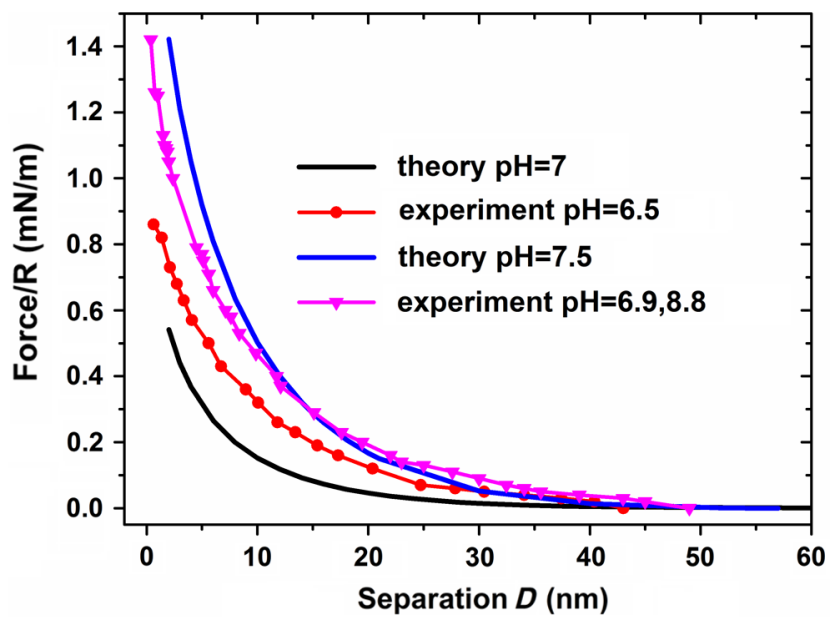

FIG. 7. Comparison between theory and experimentally measured force, both plotted as a function of distance between a colloidal particle with radius $\approx 2.5 \mu \mathrm{m}$ and Suprasil in $10^{-3} \mathrm{M}$ $\mathrm{NaNO}_{3}$ of salt solution. Experimental data are from Ref. [122]. The force has been normalized by sphere radius (in the case of the interaction between a sphere of radius $R$ and a planar surface, one has $R_{\text {eff }}=R$ ). Both the range of the force and the absolute values of the force are seen to be in good agreement. The method of force calculation is described in the text.

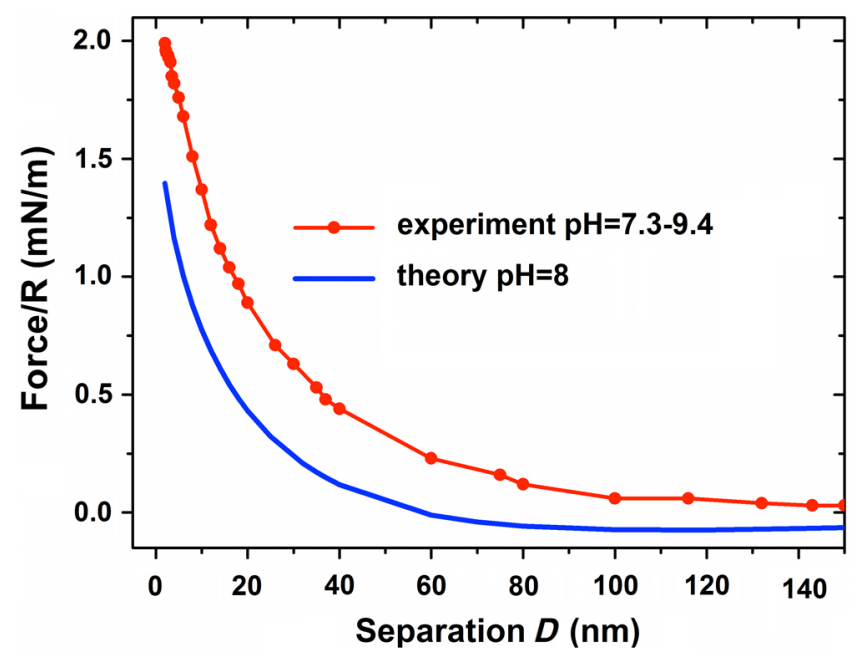

FIG. 8. Comparison between theory prediction and experimentally measured force, both plotted as a function of distance for the interaction between a radius $\approx 2.5 \mu \mathrm{m}$ spherical colloidal particle and Suprasil in $10^{-4} \mathrm{M} \mathrm{NaNO}_{3}$ of salt solution. Experimental data are from Ref. [122]. The force has been normalized by the sphere radius. Both the range of the force and the absolute value of the force are seen to be in good agreement.

Our theory can also display a similar trend observed experimentally with a variation of salt concentration and $\mathrm{pH}$ of the solution. In particular, the Debye length at $10^{-3} \mathrm{M}$ is smaller than that at $10^{-4} \mathrm{M}$; hence, the range of repulsive interaction decreases with increasing electrolyte concentration.

We have to note that there are uncertainties in the experimental measurements. For force measurements, the silica particles are usually subject to plasma cleaning treatment for a couple of minutes, which may change the surface chemistry. The experimental results often tend to overestimate the force at small separations because of elastic flattening. Besides the surface roughness, measurement technique differences can also contribute to the uncertainties of the measured results. There can also be charge reversal at high salt ion concentrations and gel layer composed of polysilicic acid formed near the surface at low $\mathrm{pH}$ [123]. In spite of such caveats, our model is clearly adequate to account for those phenomena observed in dilute electrolyte and $\mathrm{pH}$ values ranging from 5 to 9 . In particular, it is rather attractive that the good agreement with the experiments emerges naturally from first-principle equations, with no adjustable or additional parameters.

\section{THE ELECTRO-OSMOTIC EFFECT AND ITS TIME DEPENDENCE}

\section{A. Coupling to the Navier-Stokes equation}

With the surface potential trap model, the EO effect can be obtained by applying a constant electric field to the system of equations comprising the PNP equations coupled 
with the NS equation, supplemented by the incompressibility condition for the fluid. For clarity, below we present the complete set of relevant equations:

$$
\begin{gathered}
\partial n / \partial t+\boldsymbol{u} \cdot(\nabla n)+\nabla \cdot \boldsymbol{J}_{n}=0, \\
\partial p / \partial t+\boldsymbol{u} \cdot(\nabla p)+\nabla \cdot \boldsymbol{J}_{p}=0, \\
\boldsymbol{J}_{n}=-D\left(\nabla n-\frac{e}{k_{B} T} n \nabla(\psi+f+g)\right), \\
\boldsymbol{J}_{p}=-D\left(\nabla p+\frac{e}{k_{B} T} p \nabla(\psi+f+g)\right), \\
\nabla^{2} \psi=-\frac{e(p-n)}{\varepsilon}, \\
\rho_{m}\left(\frac{\partial \boldsymbol{u}}{\partial t}+\boldsymbol{u} \cdot(\nabla \boldsymbol{u})\right)=-\nabla P+\eta \nabla^{2} \boldsymbol{u} \\
-e(p-n) \nabla(\psi+f+g), \\
\nabla \cdot \boldsymbol{u}=0 .
\end{gathered}
$$

Here, $g$ denotes the electrical potential associated with the applied electric field $E$, i.e., $-\nabla g=\boldsymbol{E}$, which is parallel to the fluid-solid interface. It is to be noted that while $\psi$ is associated only with the ions [as evidenced by the righthand side of Eq. (17e)], the driving force for the ions is given by the product of the local charge density and local electric field, i.e., $-e(p-n) \nabla \varphi$, where $\varphi=\psi+f+g$. This is in contrast to the traditional treatment of the electroosmotic effect in which the driving force in the NS equation is usually expressed as $-e(p-n) \nabla g$, i.e., decoupled from the electrical potential of the ions. There is a recent exception in which the local electric field is used [124] in conjunction with the PB equation for the study of the EO effect. However, such a combination can lead to the violation of the Onsager relation (see Sec. VIII).

\section{B. Definition of the time-dependent model}

To illustrate the EO effect, consider a cylindrical sample with the relevant sections as shown in Fig. 9. Owing to the much larger amount of required computational resources, for the time-dependent simulations we consider a smaller channel radius of $a=0.48 \mu \mathrm{m}$. The surface potential trap used, over the whole region $\mathrm{A}$ to $\mathrm{D}$, has the same parameters as that shown in Fig. 2 (hence, the chemical potential is $-76.8 \mathrm{mV}$ if the channel radius is larger than $3 \mu \mathrm{m})$. To facilitate numerical convergence, however, we

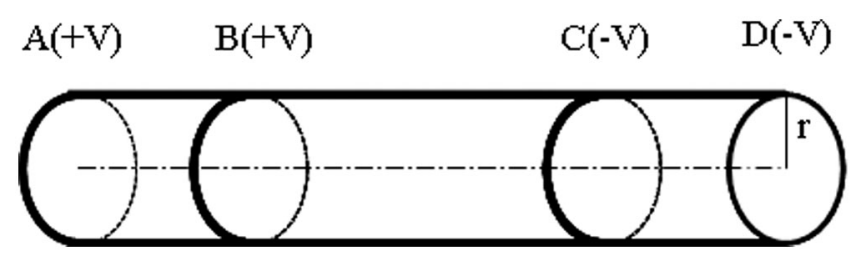

FIG. 9. Geometry of the EO-effect simulation.

have broadened the surface potential width to $0.96 \mathrm{~nm}$ in this case. An electric field $E_{z}=1.04 \times 10^{3} \mathrm{~V} / \mathrm{cm}$ is turned on abruptly at $t=0$, at the section denoted BC in Fig. 9, to simulate the EO pump, while the $\mathrm{AB}$ and $\mathrm{CD}$ sections are the inlet and outlet, respectively. The same voltage $+V$ is applied at the cross-sectional areas $\mathrm{A}$ and $\mathrm{B}$, and $-V$ is applied at the cross-sectional areas $\mathrm{C}$ and $\mathrm{D}$. Here, $V=E l$, with $2 l$ being the length of the $\mathrm{BC}$ segment. A uniform voltage at a given cross section of the cylindrical channel can be physically realized, for example, by using a fine mesh electrode fabricated with very thin metallic wires so that fluid can pass through the mesh with minimal drag. The relevant parameters are taken in accordance with those for deionized water: $D=9.32 \times 10^{-9} \mathrm{~m}^{2} / \mathrm{s}$, $n^{\infty}=9.5 \times 10^{20} / \mathrm{m}^{3}, \eta=1 \mathrm{cP}, \rho_{m}=1 \mathrm{~g} / \mathrm{cm}^{3}, \varepsilon=\varepsilon_{r} \varepsilon_{o}$ with $\varepsilon_{r}=80, \quad \varepsilon_{o}=8.85 \times 10^{-12} \mathrm{~F} / \mathrm{m}, \quad T=300 \mathrm{~K}$, $e=1.6 \times 10^{-19} \mathrm{C}$, and $k_{B}=1.38 \times 10^{-23} \mathrm{~J} / \mathrm{K}$. In the calculation, $l=\mathrm{AB}=\mathrm{CD}=\mathrm{BC} / 2=0.72 \mu \mathrm{m}$. In reference to Fig. 9, $\mathrm{V}$ is set at $75 \mathrm{mV}$. Periodic boundary conditions are applied at $\mathrm{A}$ and $\mathrm{D}$ for $\nabla g, f, \psi$, and densites $p$ and $n$.

Since AD is the sample length, the effective average electric field over this length is $520 \mathrm{~V} / \mathrm{cm}$, half the amount over the BC section. We set the potential $\psi=g$ at the fluidsolid interface, $r=a$. Other conditions are $\boldsymbol{u}=0$ at the fluid-solid interface, and $\boldsymbol{J}_{n} \cdot \boldsymbol{n}=0, \boldsymbol{J}_{p} \cdot \boldsymbol{n}=0$, where $\boldsymbol{n}$ denotes the interfacial normal.

For numerical calculations, we used the time discrete, implicit Euler scheme, which allows a larger time step. The finite element method was used for space discretization, with a piecewise linear element for the potential and charge densities. For the Stokes equation, a mixed type P2P1 element was used, which means a quadratic element for velocity and a piecewise linear element for pressure. In order to deal with nonlinear coupling, a convex iteration method was applied. Details of the numerical scheme will be published elsewhere.

It should be mentioned that in the literature, a scenario is often delineated in which there can be a region of large viscosity $[47,125]$ (the stagnant layer) near the fluid-solid interface, so the effective hydrodynamic boundary may be at the abrupt interface between the region with large viscosity and the fluid (i.e., the slip plane). There can be some consequences of having a stagnant layer. For example, if the zeta potential is measured by extrapolating the fluid velocity (through the Smoluchowski velocity formula) back to the hydrodynamic boundary, then the implied 

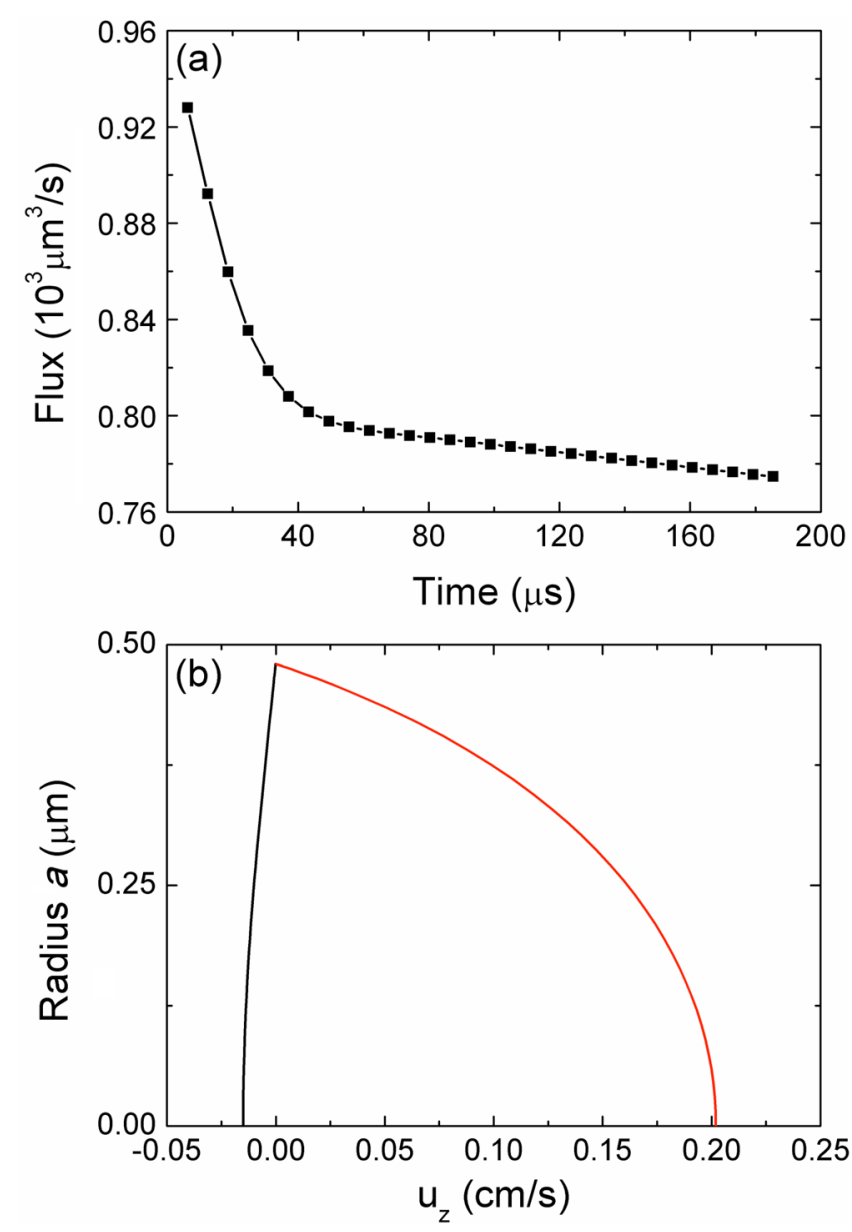

FIG. 10. The EO effect, calculated with a step-function electrical field. (a) The time dependence of the EO effect, showing a pulse of fluid flux followed by a quick decay to a smaller value. (b) The axial velocities of the positive (red line) and negative (black line) charges, at $t=182 \mu \mathrm{s}$. It is seen that the trapped surface charges essentially remain stationary, mainly owing to the nonslip hydrodynamic boundary condition. Hence, it resembles a Stern layer. However, the application of the Navier boundary condition for the NS equation can modify the picture. Here, the red line is obtained as the solution of the NS equation with $-e p \nabla \varphi$ as the driving force density, whereas the black line is that with $+e n \nabla \varphi$ as the driving force density. Since $n$ has a small value at the center of the channel, the forcing term is small, and hence the related velocity is small as well. It is seen that the sum of the two curves yields a positive EO flow.

surface charge density can be lower than that obtained by titration experiments [47]. In this work, we regard the fluid as having uniform viscosity. However, the fluid layer next to the solid, e.g., the Stern layer, may still be stagnant by virtue of the nonslip hydrodynamic boundary condition. In that case, the potential difference between the fluid-solid interface and the outer Helmholtz plane would be minimal.

\section{Electro-osmotic effect and its time dependence}

In Fig. 10, we show the existence of the EO effect, starting immediately after the application of the electric filed. There is an extremely fast initial reaction to the electric field, followed by a slower decay with a time scale that is on the order of $30 \mu s$. This time dependence is definitely not based on the inertial effect, since at this flow rate, the inertial effect is still negligible. Instead, it is a manifestation that, in response to the initial disturbance, there is a diffusive countercurrent trying to establish a new equilibrium. The initial reaction time is governed by the viscosity of the fluid, as well as the size of the system. The initial flow velocity, averaged over the cross section, is around $1.31 \mathrm{~mm} / \mathrm{s}$.

This initial flow velocity can be compared with the traditional EO-effect calculation, involving the solution of the PB equation coupled with the Stokes equation in the decoupling approximation, i.e., only with the externally applied electric field as the forcing term, as shown in Appendix A. In that case, the average flow velocity (with no time dependence) is given by the expression $(\varepsilon \zeta / \eta) E_{z}-$ $\left(a^{2} / 8 \eta\right)(-d P / d z)[73,74]$, where the (averaged) pressure gradient is along the axial direction, and the $\zeta$ potential $(=-29.8 \mathrm{mV})$ is defined by Eq. (15) by using as the boundary value $-\mu(a \rightarrow \infty)=-76.8 \mathrm{mV}$. For the parameters relevant to the present case and $d P / d z=0$, we obtain the averaged flow velocity of $1.1 \mathrm{~mm} / \mathrm{s}$.

In Fig. 10(b), we plot the axial velocities of the screening positive ions (red curve) and the associated negative ions (black curve). Here, the two curves are obtained by separately using the positive ion density or negative ion density (both obtained by first solving the coupled PNP + NS equations numerically) in the NS equation and calculating the resulting $\boldsymbol{u}^{ \pm}$, i.e.,

$$
\begin{aligned}
\rho_{m}\left(\frac{\partial \boldsymbol{u}^{ \pm}}{\partial t}+\boldsymbol{u}^{ \pm} \cdot\left(\nabla \boldsymbol{u}^{ \pm}\right)\right)= & -\nabla P+\eta \nabla^{2} \boldsymbol{u}^{ \pm} \\
& -z e\left(\begin{array}{c}
p \\
-n
\end{array}\right) \nabla \varphi .
\end{aligned}
$$

Since the system is charge neutral overall, the net integrated force density on the fluid must be zero. Hence, the EO effect arises from the difference in the flows resulting from the body forces exerted by the screening ions (red) and the counter flow (black). Owing to the hydrodynamic nonslip boundary condition, the adsorbed layer has to remain stationary. Hence, it resembles the traditional "Stern layer" in the static case as mentioned earlier.

In this picture, the momentum associated with the fluid's center-of-mass motion is counterbalanced with that of the solid boundary, which may be regarded as having infinite mass. However, either by using the Navier hydrodynamic boundary condition (i.e., by allowing a slip at the fluidsolid interface $[47,125]$ ) or by considering the movement of a spherical particle with a surface potential, i.e., electrophoresis, our model can imply significantly altered physical pictures. In particular, for the case of the Navier boundary condition, there is a slip length $\ell_{s}$, with $\ell_{s}=0$ denoting no 
slip and $\ell_{s} \rightarrow \infty$ implying total decoupling from the solid boundary. It is plausible that there should be a value of $\ell_{s}$ at which the EO flux reaches a peak. This is because a small but nonzero $\ell_{s}$ should enhance the EO flux, but an infinite $\ell_{s}$ would mean that the fluid's center of mass cannot move, owing to zero net force on the fluid and its total decoupling from the solid. Hence, there should be a peak in between. These issues will be addressed separately.

The time dependence of the EO effect is an inevitable consequence of Eq. (17), as illustrated in Fig. 10(a). This should not be surprising since the existence of diffusion means that there is always a tendency to establish a new equilibrium after the initial disturbance. This time variation should be contrasted with the traditional picture that the time-dependent EO is a consequence of the inertial effect, which is small. In experiments, it is often observed that the flow rate can decay with time [73,74]. This is partly due to electrode screening by the ions, so the applied voltage seen by the sample actually decreases with time. However, the time dependence that arises from Eq. (17) is separate from, and in addition to, the electrode screening effect. It is an intrinsic manifestation of the EO mechanism. Initially, when the electric field is abruptly turned on, a convective current will induce a flow. This is the initial pulse seen in Fig. 10(a). However, as the flow inevitably shifts the spatial pattern of charge density and the associated electrical potential, there can be a diffusive countercurrent trying to establish a new equilibrium. Thus, the whole process

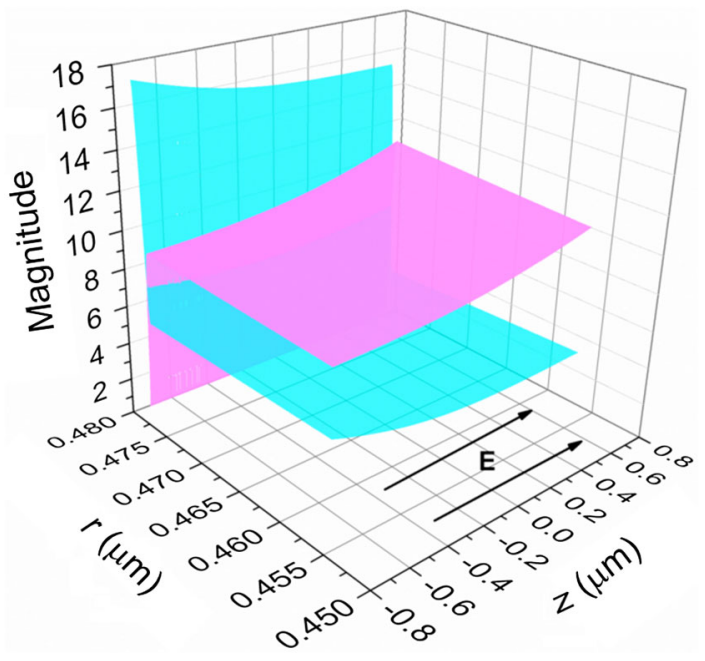

FIG. 11. The distribution of the positive ions (pink) and negative ions (blue) in the central BC section of the cylindrical channel (see Fig. 9), with a radius of $0.48 \mu \mathrm{m}$. Here, the red indicates positive ions and blue the negative ions. The magnitude is evaluated as $\ln \left[1+\right.$ density $\left./\left(950 \mu \mathrm{m}^{-3}\right)\right]$. This distribution is at $t=182 \mu$ s, i.e., at the end of the slowly varying region, as shown in Fig. 10(a). The direction of the externally applied electric field is as shown. The charge densities are seen to be biased by the external field. Along the $r$ direction, only a section close to the fluid-solid interface is shown. represents a transition from the initial static state, $\boldsymbol{J}_{n}, \boldsymbol{J}_{p}$, $\boldsymbol{u}=0$, to a final steady state, with a relaxation time scale on the order of $L^{2} / D$, where $L$ denotes the sample size. The very short time scale seen in our simulations can be attributed to the small size of our sample being considered.

In Fig. 11, we use a 2D color map to illustrate the ion density distributions at $t=182 \mu$ s, i.e., at the end of the slowly varying region shown in Fig. 10(a). It is seen that owing to the inevitable coupling between the applied electric field and the local field associated with the ion densities, the $p(\boldsymbol{x})$ and $n(\boldsymbol{x})$ are biased along the direction of the applied field. In this context, the initial pulse of the fluid flux can be easily identified as the fluid flow carried along by the convective flow of the ions.

An interesting question is whether there can be a back flow of fluid flux when the external field is turned off. We have carried out such a simulation, and the answer is that the flux simply decays to zero when the external field is turned off, with essentially no back flow; i.e., the flow process is time-irreversible.

Since the final state represents a smaller fluid flux, it follows that the optimal operational mode of the EO effect should be periodically pulsed $[73,74]$. However, the timedomain optimization of the EO effect represents a new direction that will be pursued separately.

\section{ONSAGER RELATION}

Linear response dictates that the electric current density $\boldsymbol{J}_{e}$ and the fluid current density $\boldsymbol{J}_{f}$ be linearly related to the voltage gradient $\nabla \varphi$ and the pressure gradient $\nabla P$ :

$$
\left[\begin{array}{l}
\boldsymbol{J}_{e} \\
\boldsymbol{J}_{f}
\end{array}\right]=-\left(\begin{array}{ll}
L_{11} & L_{12} \\
L_{21} & L_{22}
\end{array}\right)\left[\begin{array}{l}
\nabla \varphi \\
\nabla P
\end{array}\right]
$$

where $L_{11}$ is the electrical conductivity and $L_{22}$ is the hydrodynamic permeability. The Onsager relation states that the response matrix must be symmetric [126,127], i.e., the cross coefficients $L_{12}=L_{21}$ in physical systems. It is imperative that we examine the Onsager relation for the PB equation with the constant $\zeta$ potential boundary condition, coupled with the Navier-Stokes equation, and compare that with the PNP equations with a surface potential trap at the interface, also coupled with the Navier-Stokes equation. Consistency with the Onsager relation [74] would reflect conformation with the linear response dynamics that is common to a diverse array of physical systems.

Since the EO effect is time dependent, we restrict ourselves to the instant at $t=0^{+}(0.04 \mu \mathrm{s}$ in actual calculations). Here, $L_{21}=\left(Q / \pi a^{2}\right) /(E), Q$ being the volumetric flow rate, and $L_{12}=\left(I / \pi a^{2}\right) /(\Delta P / l)$, where $I$ denotes the electrical current and $\Delta P / l$ the cross-sectional averaged pressure gradient measured across the BC segment (Fig. 9). Here, the units of $L_{21}$ are $\left(\mathrm{m}^{2} / \mathrm{V} \cdot \mathrm{s}\right)$, which is the same as the units of $L_{12},(\mathrm{~A} / \mathrm{Pa} \cdot \mathrm{m})$. By using the calculated data only in the initial instant, we are able to 
confine our considerations to within the $\mathrm{BC}$ segment. This treatment is noted to differ from the usual EO-effect calculation, which should include the whole AD segment, and in a sense represents a minimal version of the Onsager relation. For the present case, the calculated results, for the PNP equations coupled with the NS equation and a surface potential trap at the interface, yield $L_{21}=L_{12}=$ $2.44 \times 10^{-8}\left(\mathrm{~m}^{2} / \mathrm{V} \cdot \mathrm{s}\right)$.

For the case of the PB equation, by decoupling the applied field from the local electric field associated with the ions (as usually assumed) in the Stokes equation, it is easy to show analytically (see Appendix A) that $L_{21}=L_{12}=$ $-\varepsilon \zeta / \eta=2.11 \times 10^{-8}\left(\mathrm{~m}^{2} / \mathrm{V} \cdot \mathrm{s}\right)$ in the present case, with a $\zeta$ [defined by Eq. (15)] potential value of $-29.8 \mathrm{mV}$, obtained by using $-\mu(a \rightarrow \infty)=-76.8 \mathrm{mV}$ as the boundary condition. However, if the decoupling approximation leads to consistency with the Onsager relation, then it would be a great coincidence if the use of a local electric field $(-\nabla \varphi)$ in the Stokes equation leads to the same consistency. Indeed, if instead of the decoupling approximation one uses the local electric field, then numerical evaluation leads to around a $\sim 20 \%$ discrepancy between $L_{21}$ and $L_{12}$. This discrepancy is easily understandable because if one uses the full local electric field in the Stokes equation and couples that to the PB equation, then the potential profile will be altered along the flux direction in the EO case (as shown in Fig. 11). However, for the reverse SP case, one applies only a pressure gradient (or a body force density), and hence the potential profile is uniform along the flux direction since the fluid flow is not coupled to the electrical potential in this case. The two cases, EO and SP, now refer to two different physical settings; their Onsager coefficients naturally differ as a result. In this context, it should be noted that for the PNP case, the fluid velocity $\boldsymbol{u}$ is inherently coupled to the ion potential $\psi$ via the $\boldsymbol{u} \cdot(\nabla n)$ and $\boldsymbol{u} \cdot(\nabla p)$ terms in Eqs. (17a) and (17b).

\section{SUMMARY AND CONCLUDING REMARKS}

We summarize the main points of this work as follows.

(1) The PB equation is inconsistent with the PNP equations. The consistency requirement leads to the CCPB equation, which guarantees charge neutrality. CCPB cannot generate EDL through either the Dirichlet or the Neumann type of boundary condition.

(2) A crucial element of our model is the introduction of a surface potential trap that attributes an energy cost to the interfacial charge dissociation process. The surface potential model enables the generation of a surface charge density layer and the attendant EDL, both within the computational domain. As the surface charge layer is coupled to the bulk, its magnitude can vary with the channel radius and/or bulk ion density.
(3) A reformulation of the CCPB leads to the definition of a chemical potential that arises from the constraint of overall charge neutrality. The CCPB provides a framework for the simultaneous, self-consistent determination of the chemical potential and the surface charge density.

(4) The CCPB can be reduced to the form of the PB equation, with the negative of the chemical potential serving as the electrical boundary condition. The zeta potential is defined as the quantity that drives the EK effects. It has the same value as the (negative) chemical potential in the large fluid-channel limit, but the two deviate from each other as the fluidchannel width diminishes.

(5) The predictions of the PB (CP), Gouy-Chapman (CC), and CCPB (same as the static limit of the PNP equations) models all agree in the large channel width limit. But they differ when the fluid-channel size approaches the nanometer scale.

(6) Predicted zeta potential variations with (added) salt ion concentration are in good agreement with the experiments, ranging from the nanoscale to microscale fluid-channel width.

(7) The predicted force between two charged surfaces is in good agreement with the experiments, both in terms of the magnitude and the range.

(8) With overall charge neutrality, the net force exerted by an external electric field is zero. Hence, the EO effect represents the difference in the flows induced by the screening ions and the interfacial counterions.

(9) There is an intrinsic time dependence of the EO effect that arises from the transition from the initial equilibrium state to a final steady state, with a relaxation process bridging the two.

(10) It follows from (9) that an optimal EO effect may be obtained by a periodically pulsed electric field.

(11) The Onsager relation can be used as a reality check for the various models. The PNP equations, coupled with the NS equation with a surface potential as the boundary condition, are shown to satisfy the Onsager relation.

It is important to note that further-refined, physically meaningful models may have to include a steric repulsion effect for the ions [128], especially in the high ion concentration regime $[129,130]$, the effects arising from interfacial inhomogeneities, as well as a chemical effect at the fluid-solid interface [131]. However, the consideration of these additional effects must be based on a consistent electrical framework, which is the main theme of this work. The perspectives present here should therefore be regarded as a first step towards a consistent and more inclusive model of electrokinetics. 


\section{ACKNOWLEDGMENTS}

P.S. wishes to acknowledge the support of SRFI11/ SC02 and RGC Grant No. HKUST604211 for this work. $\mathrm{He}$ also wishes to thank E. Charlaix for a helpful discussion. C. L. wishes to acknowledge partial support by NSF Grants No. DMS-1109107, No. DMS-1216938, and No. DMS-1159937. S. X. wishes to thank Long Chen and Ming Wang for help in coding, and to acknowledge China Scholarship Council for support. Li Wan and Shixin Xu contributed equally to this work.

\section{APPENDIX A: ELECTRO-OSMOTIC FLOW AND ONSAGER COEFFICIENTS FROM THE POISSON-BOLTZMANN EQUATION}

In this appendix we present a simple derivation of axial velocity in a cylindrical channel by using the PoissonBoltzmann equation, coupled with the Navier-Stokes equation. An electric field $E_{z}=-\nabla g$ is applied along the axial direction to drive the flow. Here, the applied electric field is assumed to not cause any variation in the ionic density distributions (which is actually incorrect as shown in Fig. 11). This is denoted the decoupling approximation. We solve for the steady-state solution under the condition that the ion density distribution profile along the $z$ direction remains constant.

In the steady state, the velocity normal to the axis is zero, with $u_{r}=0$. The axial velocity $u_{z}$ in the steady state can be written as

$$
\frac{\partial^{2} u_{z}}{\partial r^{2}}+\frac{1}{r} \frac{\partial u_{z}}{\partial r}=\frac{1}{\eta} \frac{\partial P}{\partial z}-\rho \frac{E_{z}}{\eta}
$$

where $\eta$ is the fluid viscosity and $P$ the pressure. The net ion charge density $\rho$ is related to the electrical potential $\psi^{(\mathrm{PB})}$ via the Poisson equation:

$$
\frac{\partial^{2} \psi^{(\mathrm{PB})}}{\partial r^{2}}+\frac{1}{r} \frac{\partial \psi^{(\mathrm{PB})}}{\partial r}=-\frac{\rho}{\varepsilon},
$$

with $\varepsilon$ being the dielectric constant. Substituting the lefthand side of Eq. (A2) into Eq. (A1) yields

$$
\frac{\partial^{2} u_{z}}{\partial r^{2}}+\frac{1}{r} \frac{\partial u_{z}}{\partial r}=\frac{1}{\eta} \frac{\partial P}{\partial z}+\frac{\varepsilon E_{z}}{\eta}\left[\frac{\partial^{2} \psi^{(\mathrm{PB})}}{\partial r^{2}}+\frac{1}{r} \frac{\partial \psi^{(\mathrm{PB})}}{\partial r}\right]
$$

The solution of Eq. (A3), for $u_{z}$, can be expressed in terms of $\psi^{(\mathrm{PB})}$ as

$$
u_{z}=-\frac{\varepsilon E_{z}}{\eta}\left[(-\mu)-\psi^{(\mathrm{PB})}\right]+\frac{a^{2}-r^{2}}{4 \eta}\left(-\frac{d P}{d z}\right)
$$

Here, $-\mu$ is the boundary value of the potential and $a$ is the channel radius, but for the traditional PB model, of course, the boundary value should be that at the infinite $a$ limit. The average axial velocity can be calculated in terms of the solution potential profile:

$$
\overline{u_{z}}=\frac{1}{a^{2}} \int_{0}^{a} 2 u_{z} r d r=-\frac{2 \varepsilon E_{z}}{\eta} \int_{0}^{1}\left[(-\mu)-\psi^{(\mathrm{PB})}\right]\left(\frac{r}{a}\right) d\left(\frac{r}{a}\right)=-\frac{\varepsilon E_{z}}{\eta} \zeta,
$$

with $d P / d z=0$. It is seen that $\bar{u}_{z}$ is proportional to the $\zeta$ potential as defined by Eq. (13).

The linear response theory states that in a dissipative system, the current density $\boldsymbol{J}_{e}$ and volume flow rate $\boldsymbol{J}_{f}$ can be linearly induced by the electric field $E_{z}$ and pressure gradient $(-d P / d z)$ :

$$
\left[\begin{array}{l}
\boldsymbol{J}_{e} \\
\boldsymbol{J}_{f}
\end{array}\right]=\left[\begin{array}{ll}
L_{11} & L_{12} \\
L_{21} & L_{22}
\end{array}\right]\left[\begin{array}{c}
E_{z} \\
-\frac{d P}{d z}
\end{array}\right]
$$

Since $\boldsymbol{J}_{f}=\int_{0}^{a} 2 u_{z} r d r / a^{2}$ and $\boldsymbol{J}_{e}=\int_{0}^{a} 2 \rho u_{z} r d r / a^{2}$, we have

$$
\begin{gathered}
L_{12}=\frac{1}{2 \eta a^{2}} \int_{0}^{a}\left(a^{2}-r^{2}\right) \rho r d r, \\
L_{21}=-\frac{2 \varepsilon}{\eta a^{2}} \int_{0}^{a}\left((-\mu)-\psi^{(\mathrm{PB})}\right) r d r .
\end{gathered}
$$

Using Eq. (A2) to express $\rho$ in Eq. (A7) yields 


$$
\begin{aligned}
L_{12} & =-\frac{\varepsilon}{2 \eta a^{2}} \int_{0}^{a}\left(a^{2}-r^{2}\right)\left[\frac{\partial^{2} \psi^{(\mathrm{PB})}}{\partial r^{2}}+\frac{1}{r} \frac{\partial \psi^{(\mathrm{PB})}}{\partial r}\right] r d r \\
& =-\frac{\varepsilon}{2 \eta a^{2}}\left\{\left[2 a^{2}(-\mu)+\psi^{(\mathrm{PB})}(0) a^{2}-\int_{0}^{a} 6 r \psi^{(\mathrm{PB})} d r\right]+\left[-\psi^{(\mathrm{PB})}(0) a^{2}+\int_{0}^{a} 2 r \psi^{(\mathrm{PB})} d r\right]\right\} \\
& =-\frac{\varepsilon}{2 \eta a^{2}}\left[2 a^{2}(-\mu)-\int_{0}^{a} 4 r \psi^{(\mathrm{PB})} d r\right] \\
& =-\frac{2 \varepsilon}{\eta a^{2}} \int_{0}^{a}\left((-\mu)-\psi^{(\mathrm{PB})}\right) r d r .
\end{aligned}
$$

Thus, the Onsager relation is satisfied, i.e., $L_{12}=L_{21}=$ $-\varepsilon \zeta / \eta$ in the decoupling approximation, where we have used the definition of the $\zeta$ potential as given in Eq. (15).

\section{APPENDIX B: VARIATIONAL DERIVATION OF THE CHEMICAL POTENTIAL}

We would like to minimize the free energy of the ions and their electrostatic interaction energy, which can be expressed as

$$
F=k_{B} T \int_{\Omega} d x\left(n \ell n \frac{n}{n^{\infty}}+p \ell n \frac{p}{n^{\infty}}+\frac{\varepsilon}{2 k_{B} T}|\nabla \varphi|^{2}\right) .
$$

The charge-neutrality constraint can be applied by using the chemical potential $\mu$ as a Lagrange multiplier. Hence,

$$
\begin{aligned}
F= & k_{B} T \int_{\Omega} d x\left(n \ell n \frac{n}{n^{\infty}}+p \ell n \frac{p}{n^{\infty}}+\frac{\varepsilon}{2 k_{B} T}|\nabla \varphi|^{2}\right) \\
& +\mu e \int_{\Omega} d x(p-n) .
\end{aligned}
$$

The electrostatic term may be integrated by parts to yield the form of a $\varphi \nabla^{2} \varphi$ term. Then, by using the Poisson equation as well as the Green function solution of $\varphi$,

$$
\varphi(\boldsymbol{x})=\frac{e}{\varepsilon} \int_{\Omega} G(\boldsymbol{x}, \boldsymbol{y})(n-p)(\boldsymbol{y}) d \boldsymbol{y},
$$

we obtain the following expression for $F$ :

$$
\begin{aligned}
F= & k_{B} T \int_{\Omega} d \boldsymbol{x}\left(n \ell n \frac{n}{n^{\infty}}+p \ell n \frac{p}{n^{\infty}}\right) \\
& +\frac{e}{2 \varepsilon} \int_{\Omega} \int_{\Omega} d \boldsymbol{x} d \boldsymbol{y}(p-n)(\boldsymbol{x}) G(\boldsymbol{x}, \boldsymbol{y})(n-p)(\boldsymbol{y}) \\
& +\mu e \int_{\Omega} d \boldsymbol{x}(p-n) .
\end{aligned}
$$

By taking the variation of $F$ with respect to $n$ and $p$ and setting it to zero, one obtains

$$
\begin{aligned}
& \frac{\delta F}{\delta n}=k_{B} T\left(1+\ell n \frac{n}{n^{\infty}}\right)-e \varphi+e \mu=0, \\
& \frac{\delta F}{\delta p}=k_{B} T\left(1+\ell n \frac{p}{n^{\infty}}\right)+e \varphi-e \mu=0 .
\end{aligned}
$$

Solving for $n$ and $p$ and setting their volume integrals to be equal, we obtain $\mu$ as given by Eq. (10b).

[1] F. F. Reuss, Notice sur un Nouvel Effet de L'electricite Galvanique, Mem. Soc. Imp. Nat. Moscou 2, 327 (1809).

[2] R. J. Hunter, Zeta Potential in Colloid Science: Principles and Applications (Academic Press, London, 1981).

[3] L. Journiaux and J. P. Pozzi, Laboratory Measurements Anomalous 0.1-0.5 Hz Streaming Potential under Geochemical Changes: Implications for Electrotelluric Precursors to Earthquakes, J. Geophys. Res. 102, 15335 (1997).

[4] P. Reppert, F. D. Morgan, D. P. Lesmes, and L. Jouniaux, Frequency Dependent Streaming Potentials J. Colloid Interface Sci. 234, 194 (2001).

[5] P. M. Reppert and F. D. Morgan, Frequence Dependent Electroosmosis, J. Colloid Interface Sci. 254, 372 (2002). 
[6] S. Yeh, M. Seul, and B. Shraiman, Assembly of Ordered Colloidal Aggregrates by Electric-Field-Induced Fluid Flow, Nature (London) 386, 57 (1997).

[7] M. Trau, D. A. Saville, and I. A. Aksay, Assembly of Colloidal Crystals at Electrode Interfaces, Langmuir 13, 6375 (1997).

[8] C. Faure, N. Decoster, and F. Argoul, AC Field Induced Two-Dimensional Aggregation of Multilamellar Vesicles, Eur. Phys. J. B 5, 87 (1998).

[9] A. Ramos, H. Morgan, N. G. Green, and A. Castellanos, AC Electrokinetics: A Review of Forces in Microelectrode Structures, J. Phys. D 31, 2338 (1998).

[10] A. Ramos, H. Morgan, N. G. Green, and A. Castellanos, AC Electric-Field-Induced Fluid Flow in Microelectrodes, J. Colloid Interface Sci. 217, 420 (1999).

[11] N. G. Green, A. Ramos, A. González, H. Morgan, and A. Castellanos, Fluid Flow Induced by Nonuniform AC Electric Fields in Electrolytes on Microelectrodes. I. Experimental Measurements, Phys. Rev. E 61, 4011 (2000).

[12] A. González, A. Ramos, N. G. Green, A. Castellanos, and H. Morgan, Fluid Flow Induced by Nonuniform AC Electric Fields in Electrolytes on Microelectrodes. II. A Linear Double-Layer Analysis, Phys. Rev. E 61, 4019 (2000).

[13] A. Ajdari, Pumping Liquids Using Asymmetric Electrode Arrays, Phys. Rev. E 61, R45 (2000).

[14] N. G. Green, A. Ramos, and H. Morgan, AC Electrokinetics: A Survey of Sub-Micrometre Particle Dynamics, J. Phys. D 33, 632 (2000).

[15] A. B. D. Brown, C. G. Smith, and A. R. Rennie, Pumping of Water with an AC Electric Field Applied to Asymmetric Pairs of Microelectrodes, Phys. Rev. E 63, 016305 (2000).

[16] V. Studer, A. Pépin, Y. Chen, and A. Ajdari, Fabrication of Microfluidic Devices for AC Electrokinetic Fluid Pumping, Microelectron. Eng. 61-62, 915 (2002).

[17] N. G. Green, A. Ramos, A. González, H. Morgan, and A. Castellanos, Fluid Flow Induced by Nonuniform AC Electric Fields in Electrolytes on Microelectrodes. III. Observation of Streamlines and Numerical Simulation, Phys. Rev. E 66, 026305 (2002).

[18] F. Nadal, F. Argoul, P. Kestener, B. Pouligny, C. Ybert, and A. Ajdari, Electrically Induced Flows in the Vicinity of a Dielectric Stripe on a Conducting Plane, Eur. Phys. J. E 9, 387 (2002).

[19] F. Nadal, F. Argoul, P. Hanusse, B. Pouligny, and A. Ajdari, Electrically Induced Interactions between Colloidal Particles in the Vicinity of a Conducting Plane, Phys. Rev. E 65, 061409 (2002).

[20] C. Marquet, A. Buguin, L. Talini, and P. Silberzan, Rectified Motion of Colloids in Asymmetrically Structured Channels, Phys. Rev. Lett. 88, 168301 (2002).

[21] W. D. Ristenpart, I. A. Aksay, and D. A. Saville, Electrically Guided Assembly of Planar Superlattices in Binary Colloidal Suspensions, Phys. Rev. Lett. 90, 128303 (2003).

[22] M. Mpholo, C. G. Smith, and A. B. D. Brown, Low Voltage Plug Flow Pumping Using Anisotropic Electrode Arrays, Sens. Actuators B 92, 262 (2003).
[23] A. Ramos, A. González, A. Castellanos, N. G. Green, and H. Morgan, Pumping of Liquids with AC Voltages Applied to Asymmetric Pairs of Microelectrodes, Phys. Rev. E 67, 056302 (2003).

[24] W. Helfrich, Deformation of Lipid Bilayer Spheres by Electric Fields, Z. Naturforsch. C 29, 182 (1974).

[25] D. J. Harrison, Z. A. Man, Z. A. Fan, and H. Widmer, Capillary Electrophoresis and Sample Injection Systems Integrated on a Planar Glass Chip, Anal. Chem. 64, 1926 (1992).

[26] M. D. Mitov, P. Méleard, M. Winterhalter, M. I. Angelova, and P. Bothorel, Electric-Field-Dependent Thermal Fluctuations of Giant Vesicles, Phys. Rev. E 48, 628 (1993).

[27] C. S. Effenhanser, A. Paulus, Z. A. Man, and H. M. Widmer, High Speed Separation of Antisence Oligonucleotides on a Micromachined Capillary Electrophoresis Device, Anal. Chem. 66, 2949 (1994).

[28] R. Pethig, Dielectrophoresis: Using Inhomogeneous AC Electrical Fields to Separate and Manipulate Cells, Critical Rev. Biotech. 16, 331 (1996).

[29] C. Werner, U. Konig, A. Augsburg, C. Arnhold, H. Korber, R. Zimmermann, and H.-J. Jacobash, Electrokinetic Surface Characterization of Biomedical Polymers: A Survey, Colloids Surf. A 159, 519 (1999).

[30] C. Werner and H.-J. Jacobash, Surface Characterization of Polymers for Medical Devices, Int. J. Artifi. Organ. 22, 160 (1999).

[31] A. E. Herr, J. I. Molho, J. G. Santiago, M. G. Mungal, T. W. Kenny, and M. G. Garguilo, Electroosmotic Capillary Flow with Non-uniform Zeta Potential, Anal. Chem. 72, 1053 (2000).

[32] T. K. Jun and C. J. Kim, Valveless Pumping Using Traversing Vapor Bubbles in Microchannels, J. Appl. Phys. 83, 5658 (1998).

[33] L. Junghoon and K. Chang-Jin, Surface-Tension-Driven Microactuation Based on Continuous Electrowetting, J. Microelectromech. Syst. 9, 171 (2000).

[34] S. Bohm, B. Timmer, W. Olthuis, and P. Bergveld, A Closed-Loop Controlled Electrochemically Actuated Micro-Dosing System, J. Micromech. Microeng. 10, 498 (2000).

[35] A. V. Lemoff and A. P. Lee, An AC Magnetohydrodynamic Micropump, Sens. Actuators B 63, 178 (2000).

[36] S. A. Miller, V. Y. Young, and C. R. Martin, Electroosmotic Flow in Template-Prepared Carbon Nanotube Membranes, J. Am. Chem. Soc. 123, 12335 (2001).

[37] S. L. Zeng, C. H. Chen, J. C. Mikkelsen Jr., and J. G. Santiago, Fabrication and Characterization of Electroosmotic Micropumps, Sens. Actuators B 79, 107 (2001).

[38] M. J. Pikal, The Role of Electroosmotic Flow in Transdermal Tontophoresis, Adv. Drug Delivery Rev. 46, 281 (2001).

[39] L. N. Jiang, J. Mikkelsen, J.-M. Koo, D. Huber, S. Yao, L. Zhang, P. Zhou, J. G. Maveety, R. Prasher, J. G. Santiago, T. W. Kenny, and K. E. Goodson, Closed-Loop Electroosmotic Microchannel Cooling System for VLSI Circuits, IEEE Trans. Comp. Pack. Tech. 25, 347 (2002).

[40] C. H. Chen and J. G. Santiago, A Planar Electroosmotic Micropump, J. Microelectromech. Syst. 11, 672 (2002). 
[41] Y. Takamura, H. Onoda, H. Inokuchi, S. Adachi, A. Oki, and Y. Horiike, Low-Voltage Electroosmosis Pump for Stand-Alone Microfluidics Devices, Electrophoresis 24185 (2003).

[42] J.-M. Wang and L.-J. Yang, Electrohydrodynamic (EHD) Micropumps with Electrode Protection by Parylene and Gelatin, Tamkang J. Sci. Eng. 8, 231 (2005).

[43] P. Wang, Z. L. Chen, and H. C. Chang, A New ElectroOsmotic Pump Based on Silica Monoliths, Sens. Actuators B 113, 500 (2006).

[44] L. X. Chen, J. Choot, and B. Yan, The Microfabricated Electrokinetic Pump: A Potentially Promising Drug Delivery Technique, Expert Opin. Drug Delivery 4, 119 (2007).

[45] J.-Y. Miao, Z.-L. Xu, X.-Y. Zhang, N. Wang, Z.-Y. Yang, and P. Sheng, Micropumps Based on the Enhanced Electroosmotic Effect of Aluminum Oxide Membranes, Adv. Mater. 19, 4234 (2007).

[46] G. Gouy, Sur la Constitution de la Charge Electrique á la Surface d'un Electrolyte, Comt. Rend. 149, 654 (1909).

[47] J. Lyklema, Fundamentals of Interface and Colloid Science (Academic, London, 1995) Vol. 2.

[48] R. Probstein, Physicochemical Hydrodynamics (John Wiley and Sons, New York, 1994).

[49] J. S. Newman and K. E. Thomas-Alyea, Electrochemical Systems (Wiley-IEEE, New York, 2004).

[50] J. Lyklema, Fundamentals of Interface and Colloid Scienec (Academic, London, 1991) Vol. 1.

[51] A. J. Bard and L. R. Faulkner, Electrochemical Methods: Fundamentals and Applications (Wiley, New York, 1980).

[52] G. Gouy, Sur la Fonction Electrocapillaire I-III, Ann. Chim. Phys. 29, 145 (1903).

[53] G. Gouy and C. R. Hebd, Sur la Constitution de la Charge Electrique a la Surface dun Electrolyte, Seances Acad. Sci. 149, 654 (1909).

[54] G. Gouy, Sur la Constitution de la Charge Electrique a la Surface dun Electrolyte, J. Phys. Theor. Appl. 9, 457 (1910).

[55] A. Einstein, Über die von der molekularkinetischen Theorie der Wärme geforderte Bewegung von in ruhenden Flüssigkeiten suspendierten Teilchen, Ann. Phys. (Berlin) 322, 549 (1905).

[56] P. Debye and E. Hückel, The Theory of Electrolytes I. The Lowering of the Freezing Point and Related Occurrences, Phys. Z. 24, 185 (1923).

[57] P. Debye and E. Hückel, Theory of Electrolytes. Part II. Law of the Limit of Electrolytic Conduction, Phys. Z. 24, 305 (1923).

[58] D. L. Chapman, A Contribution to the Theory of Electrocapillarity, Philos. Mag. 25, 475 (1913).

[59] P. Delahay, Double Layer and Electrode Kinetics (Wiley, New York, 1965).

[60] J. O’M. Bockris and A. K. N. Reddy, Modern Electrochemistry (Plenum, New York, 1970).

[61] M. Sluyters-Rehbach and J. H. Sluyters, Electroanalytical Chemistry (Marcel Dekker, New York, 1970).

[62] R. Parsons, Electrical Double Layer: Recent Experimental and Theoretical Developments, Chem. Rev. 90, 813 (1990).
[63] O. Stern, Zur Theorie der Elektrolytischen Doppelschicht, Z. Elektrochem. 30, 508 (1924).

[64] B. M. Grafov and A. A. Chernenko, Direct Current Passing through a Binary Electrolyte Solution: Theory, Dokl. Akad. Nauk SSSR Fiz. Khim. 146, 135 (1962).

[65] A. A. Chernenko, On the Theory of Direct Current Passing through a Binary Electrolyte Solution, Dokl. Akad. Nauk SSSR Fiz. Khim. 153, 1129 (1963).

[66] J. Newman, The Polarized Diffuse Double Layer, Trans. Faraday Soc. 61, 2229 (1965).

[67] A. D. Macgillivray, Nernst Planck Equations, and the Electroneutrality, and Donnan Equilibrium Assumptions, J. Chem. Phys. 48, 2903 (1968).

[68] R. B. Whitney and D. C. Grahame, A Modified Theory of the Electrical Double Layer, J. Chem. Phys. 9, 827 (1941).

[69] S. Ghosal, Fluid Mechanics of Electroosmotic Flow and Its Effect on Band Broadening in Capillary Electrophoresis, Electrophoresis 25, 214 (2004).

[70] J. R. Macdonald, Impedance Spectroscopy: Old Problems and New Developments, Electrochim. Acta 35, 1483 (1990).

[71] L. A. Geddes, Historical Evolution of Circuit Models for the Electrode-Electrolyte Interface, Ann. Biomed. Eng. 25, 1 (1997).

[72] M. Z. Bazant, K. Thornton, and A. Ajdari, Diffuse-Charge Dynamics in Electrochemical Systems, Phys. Rev. E 70, 021506 (2004).

[73] Z. Xu, J. Y. Miao, N. Wang, W. Wen, and P. Sheng, Maximum Efficiency of the Electro-Osmotic Pump, Phys. Rev. E 83, 066303 (2011).

[74] Z. Xu, J. Y. Miao, N. Wang, W. Wen, and P. Sheng, Digital Flow Control of Electroosmotic Pump: Onsager Coefficients and Interfacial Parameters Determination, Solid State Commun. 151, 440 (2011).

[75] W. A. Ducker, T. J. Sendem, and R. M. Pashley, Direct Measurement of Colloidal Force Using an Atomic Force Microscope, Nature (London) 353, 239 (1991).

[76] P. G. Hartley, I. Larson, and P. J. Scales, Electrokinetic and Direct Force Measurements between Silica and Mica Surfaces in Dilute Electrolyte Solutions, Langmuir 13, 2207 (1997).

[77] I. Popa, P. Sinha, M. Finessi, P. Maroni, G. Papastavrou, and M. Borkovec, Importance of Charge Regulation in Attractive Double-Layer Forces between Dissimilar Surfaces, Phys. Rev. Lett. 104, 228301 (2010).

[78] F. H. J. van der Heyden, D. Stein, and C. Dekker, Streaming Currents in a Single Nanofluidic Channel, Phys. Rev. Lett. 95, 116104 (2005).

[79] K. L. Jensen, J. T. Kristensen, A. M. Crumrine, M. B. Andersen, H. Bruus, and S. Pennathur, HydroniumDominated Ion Transport in Carbon-Dioxide-Saturated Electrolytes at Low Salt Concentrations in Nanochannels, Phys. Rev. E 83, 056307 (2011).

[80] L. Bocquet and E. Charlaix, Nanofluidics, from Bulk to Interfaces, Chem. Soc. Rev. 39, 1073 (2010)

[81] A. van den Berg, H. G. Craighead, and P. Yang, From Microfluidic Applications to Nanofluidic Phenomena, Chem. Soc. Rev. 39, 899 (2010). 
[82] D. Stein, M. Kruithof, and C. Dekker, Surface-ChargeGoverned Ion Transport in Nanofluidic Channels, Phys. Rev. Lett. 93, 035901 (2004).

[83] R. P. Camara, G. Papastavrou, S. H. Behrens, and M. Borkovec, Interaction between Charged Surfaces on the Poisson-Boltzmann Level: The Constant Regulation Approximation, J. Phys. Chem. B 108, 19467 (2004).

[84] S. L. Carnie and D. Y. C. Chan, Interaction Free Energy between Plates with Charge Regulation: A Linearized Model, J. Colloid Interface Sci. 161,260 (1993).

[85] S. H. Behrens and D. G. Grier, The Charge of Glass and Silica Surfaces, J. Chem. Phys. 115, 6716 (2001).

[86] C. Gentil, D. Côte, and U. Bockelmann, Transistor Based Study of the Electrolyte/SiO2 Interface, Phys. Status Solidi (a) 203, 3412 (2006).

[87] J. C. Fogarty, H. M. Aktulga, A. Y. Grama, A. C. T. Van Duin, and S. A. Pandit, A Reactive Molecular Dynamics Simulation of the Silica-Water Interface, J. Chem. Phys., 132, 174704 (2010).

[88] T. Das, D. Bratko, L. B. Bhuiyan, and C. W. Outhwaite, Polyelectrolyte Solutions Containing Mixed Valency Ions in the Cell Model: A Simulation and Modified Poisson-Boltzmann Study, J. Chem. Phys. 107, 21 (1997).

[89] Q. Liu, Y. Wang, W. Guo, H. Ji, J. Xue, and Q. Ouyang, Asymmetric Properties of Ion Transport in a Charged Conical Nanopore, Phys. Rev. E 75, 051201 (2007).

[90] M. van Soestbergen, P. M. Bieshervel, R. T. H. Rongen, L. J. Ernst, and G. Q. Zhang, Modified PoissonNernst-Planck Theory for Ion Transport in Polymeric Electrolytes, J. Electrost. 66, 567 (2008).

[91] Y. K. Suh and S. Kang, Asymptotic Analysis of Ion Transport in a Nonlinear Regime around Polarized Electrodes under AC, Phys. Rev. E 77, 031504 (2008).

[92] Y. K. Suh and S. Kang, Numerical Prediction of $A C$ Electro-Osmotic Flows around Polarized Electrodes, Phys. Rev. E 79, 064309 (2009).

[93] M. Baptista, R. Schmitz, and B. Duenweg, Simple and Robust Solver for the Poisson-Boltzmann Equation, Phys. Rev. E 80, 016705 (2009).

[94] A. Golovnev and S. Trimper, Analytical Solution of the Poisson-Nernst-Planck Equations in the Linear Regime at an Applied DC-Voltage, J. Chem. Phys. 134, 154902 (2011).

[95] A. Golovnev and S. Trimper, Steady State Solution of the Poisson-Nernst-Planck Equations, Phys. Lett. A 374, 2886 (2010).

[96] F. Beunis, F. Strubbe, M. Marescaux, and K. Neyts, Diffuse Double Layer Charging in Nonpolar Liquids, Appl. Phys. Lett. 91, 182911 (2007).

[97] M. Marescaux, F. Beunis, F. Strubbe, B. Verboven, and K. Neyts, The Impact of Diffusion Layers in Strong Electrolytes on the Transient Current, Phys. Rev. E 79, 011502 (2009).

[98] A. E. Cárdenas, R. D. Coalson, and M. G. Kurnikova, Three-Dimensional Poisson-Nernst-Planck Theory Studies: Influence of Membrane Electrostatics on Gramicidin A Channel Conductance, Biophys. J. 79, 80 (2000).

[99] R. D. Coalson and M. G. Kurnikova, Poisson-NernstPlanck Theory Approach to the Calculation of Current through Biological Ion Channels, IEEE Trans. Nanobioscience 4, 81 (2005).

[100] D. A. Doyle, J. M. Cabral, R. A. Pfuetzner, A. Kuo, J. M. Gulbis, S. L. Cohen, B. T. Chait, and R. MacKinnon, The Structure of the Potassium Channel: Molecular Basis of $\mathrm{K}+$ Conduction and Selectivity, Science 280, 69 (1998).

[101] P. Graf, A. Nitzan, M. G. Kurnikova, and R. D. Coalson, A Dynamic Lattice Monte Carlo Model of Ion Transport in Inhomogeneous Dielectric Environments: Method and Implementation, J. Phys. Chem. B 104, 12324 (2000).

[102] P. Graf, M. Kurnikova, R. Coalson, and A. Nitzan, Comparison of Dynamic Lattice Monte Carlo Simulations and the Dielectric Self-Energy Poisson-Nernst-Planck Continuum Theory for Model Ion Channels, J. Phys. Chem. B 108, 2006 (2004).

[103] M. Kurnikova, R. D. Coalson, P. Graf, and A. Nitzan, A Lattice Relaxation Algorithm for Three-Dimensional Poisson-Nernst-Planck Theory with Application to Ion Transport through the Gramicidin A Channel, Biophys. J. 76, 642 (1999).

[104] C.-C. Lee, H. Lee, Y. K. Hyon, T.-C. Lin, and C. Liu, New Poisson-Boltzmann Type Equations: One-Dimensional Solutions, Nonlinearity 24, 431 (2011).

[105] M. Polat, K. Sato, T. Nagaoka, and K. Watari, Effect of PH and Hydration on the Normal and Lateral Interaction Forces between Alumina Surfaces, J. Colloid Interface Sci. 304, 378 (2006).

[106] B. J. Kirby and E. F. Hasselbrink, Zeta Potential of Microfluidic Substrates: 1. Theory, Experimental Techniques, and Effects on Separations, Electrophoresis 25, 187 (2004).

[107] R. K. Iler, The Chemistry of Silica (Wiley, New York, 1979).

[108] T. Hiemstra1, P. Venema, and W. H. V. Riemsdijk, Intrinsic Proton Affinity of Reactive Surface Groups of Metal (Hydr)oxides: The Bond Valence Principle, J. Colloid Interface Sci. 133, 105 (1989).

[109] M. Předota, A. V. Bandura, P. T. Cummings, J. D. Kubicki, D. J. Wesolowski, A. A. Chialvo, and M. L. Machesky, Electric Double Layer at the Rutile (110) Surface. 1. Structure of Surfaces and Interfacial Water from Molecular Dynamics by Use of AB Initio Potentials, J. Phys. Chem. B 108, 12049 (2004).

[110] M. Sulpizi, M. P. Gaigeot, and M. Sprik, The Silica-Water Interface: How the Silanols Determine the Surface Acidity and Modulate the Water Properties, J. Chem. Theory Comput. 8, 1037 (2012).

[111] M. P. Gaigeot, M. Sprik, and M. Sulpizi, Oxide/ Water Interfaces: How the Surface Chemistry Modifies Interfacial Water Properties, J. Phys. Condens. Matter 24, 124106 (2012).

[112] J. E. Melanson, N. E. Baryla, and C. A. Lucy, Dynamic Capillary Coatings for Electroosmotic Flow Control in Capillary Electrophoresis. TrAC, Trends Anal. Chem. 20, 365 (2001).

[113] P. Somasundaran, T. W. Healy, and D. W. Fuerstenau, Surfactant Adsorption at the Solid-Liquid InterfaceDependence of Mechanism on Chain Length, J. Phys. Chem. 68, 3562 (1964). 
[114] J. Westall and H. Hohl, Comparison of Models for the Oxide-Solution Interface, Adv. Colloid Interface Sci. 12, 265 (1980).

[115] T. Hiemstra, J. C. M. de Wit, and W. H. van Riemsdijk, Multisite Proton Adsorption Modeling at the Solid/ Solution Interface of (Hydr)oxides: A New Approach: II. Application to Various Important (Hydr)oxides, J. Colloid Interface Sci. 133, 105 (1989).

[116] P. J. Scales, F. Grieser, and T. W. Healy, Electrokinetics of the Silica-Solution Interface: A Flat Plate Streaming Potential Study, Langmuir 8, 965 (1992).

[117] C. Berli, M. V. Piaggio, and J. A. Deiber, Modeling the Zeta Potential of Silica Capillaries in Relation to the Background Electrolyte Composition, Electrophoresis 24, 1587 (2003).

[118] A. Plecis, R. B. Schoch, and P. Renaud, Ionic Transport Phenomena in Nanofluidics: Experimental and Theoretical Study of the Exclusion-Enrichment Effect on a Chip, Nano Lett. 5, 1147 (2005).

[119] R. B. Schoch, J. Y. Han, and P. Renaud, Transport Phenomena in Nanofluidics, Rev. Mod. Phys. 80, 839 (2008).

[120] F. H. J. van der Heyden, D. Stein, K. Besteman, S. G. Lemay, and C. Dekker, Charge Inversion at High Ionic Strength Studied by Streaming Currents, Phys. Rev. Lett. 96, 224502 (2006).

[121] C. M. Cerda, Mobilization of Quartz Fines in Porous Media, Clays Clay Miner. 36, 491 (1988).

[122] P. G. Hartley, I. Larson, and P. J. Scales, Electrokinetic and Direct Force Measurements between Silica and Mica Surfaces in Dilute Electrolyte Solutions, Langmuir 13, 2207 (1997).
[123] G. Vigil, Z. Xu, S. Steinberg, and J. Israelachvili, Interactions of Silica Surfaces, J. Colloid Interface Sci. 165, 367 (1994).

[124] M. Polat, K. Sato, T. Nagaoka, and K. Watari, Comparison of the Nernst-Planck Model and the Poisson-Boltzmann Model for Electroosmotic Flows in Microchannels, J. Colloid Interface Sci. 315, 731 (2007).

[125] J. Lyklema, On the Slip Process in Elelctrokinetics, Colloids Surf. A 92, 41 (1994).

[126] L. Onsager, Reciprocal Relations in Irreversible Processes. II. Phys. Rev. 38, 2265 (1931).

[127] L. Onsager, Reciprocal Relations in Irreversible Processes. I., Phys. Rev. 37, 405 (1931).

[128] M. Z. Bazant, M. S. Kilic, B. Storey, and A. Ajdari, Towards an Understanding of Induced-Charge Electrokinetics at Large Applied Voltages, Adv. Colloid Interface Sci. 152, 48 (2009).

[129] S. Das and S. Hardt, Steric-Effect-Induced Enhancement of Electrical-Double-Layer Overlapping Phenomena, Phys. Rev. E 84, 012501 (2011).

[130] V. L. Shapovalov and G. Brezesinski, Breakdown of the Gouy-Chapman Model for Highly Charged Langmuir Monolayers: Counterion Size Effect, J. Phys. Chem. B 110, 10032 (2006).

[131] Z. Zhang, P. Fenter, L. Cheng, N. C. Sturchio, M. J. Bedzyk, M. Predota, O. A. Bandura, J. D. Kubicki, S. N. Lvov, P. T. Cummings, A. A. Chialvo, M. K. Ridley, P. Benezeth, L. Anovitz, D. A. Palmer, M. L. Machesky, and D. J. Wesolowski, Ion Adsorption at the Rutile-Water Interface: Linking Molecular and Macroscopic Properties, Langmuir 20, 4954 (2004). 